\title{
POPULATION GENETICS MODELS WITH SKEWED FERTILITIES: A FORWARD AND BACKWARD ANALYSIS
}

\author{
THIERRY HUILLET, MARTIN MÖHLE
}

\begin{abstract}
Discrete population genetics models with unequal (skewed) fertilities are considered, with an emphasis on skewed versions of Cannings models, conditional branching process models in the spirit of Karlin and McGregor, and compound Poisson models. Three particular classes of models with skewed fertilities are investigated, the Wright-Fisher model, the Dirichlet model, and the Kimura model. For each class the asymptotic behavior as the total population size $N$ tends to infinity is investigated for power law fertilities and for geometric fertilities. This class of models can exhibit a rich variety of sub-linear or even constant effective population sizes. Therefore, the models are not necessarily in the domain of attraction of the Kingman coalescent. For a substantial range of the parameters, discrete-time coalescent processes with simultaneous multiple collisions arise in the limit.
\end{abstract}

Running title: Population genetics models with skewed fertilities.

Keywords: Ancestral process; Cannings model; Compound Poisson model; Dirichlet model; Dirichlet-Kingman coalescent; Duality; Evolutionary processes; Exchangeable coalescent; Karlin and McGregor model; Kimura model; Kingman coalescent; Population dynamics; Simultaneous multiple collisions; Wright-Fisher model

\section{INTRODUCTION}

The well-known neutral discrete Wright-Fisher model describes the evolution of a population of constant size $N$ from generation to generation forwards in time. One of the main features of this model (and similar models such as the discrete Moran model) is that each individual has the same propensity to produce offspring.

In this paper discrete population models are studied, in which individuals may have unequal propensities to reproduce. We shall speak of models with skewed fertilities. The terminology 'skewed' means here 'asymmetric' or 'unequal' and should be not confused with the skewness of a distribution. These models turn out to be of interest mainly because of the following two reasons. First of all, it turns out that the effective population size $N_{e}$ (a precise definition of $N_{e}$ is provided after Eq. (8)) of these models may differ significantly from the actual total population size $N$ and may not even depend linearly on $N$. The fact that models with this behavior may have importance for biological applications, is for example indicated in papers of Eldon and Wakeley [6] and Wakeley and Sargsyan [34]. Models with effective population sizes smaller than $N$ occur also in a different context of age-structure of populations in a paper of Sagitov and Jagers [30]. Secondly, in the limit as the total population size $N$ tends to infinity, these models are not necessarily in

Date: April 5, 2011. 
the domain of attraction (see Definition 2.1) of the Kingman coalescent [14]. The limiting coalescent may have simultaneous multiple collisions of ancestral lineages. Ancestral processes of this form are a major research area in coalescent theory and have been studied intensively since at least ten years (see, for example, [24] or [31]).

The models we are mainly interested in are obtained by conditioning a sequence $\xi_{1}, \xi_{2}, \ldots$ of independent random variables on the event that $\xi_{1}+\cdots+\xi_{N}=N$, with the interpretation that the outcome of $\xi_{n}$, under the constraint that $\xi_{1}+$ $\cdots+\xi_{N}=N$, is the number of children of the $n$th individual, $n \in\{1, \ldots, N\}$. Since the random variables $\xi_{1}, \xi_{2}, \ldots$ are not necessarily assumed to be identically distributed, this construction results in unequal propensities of the individuals to produce offspring. In the spirit of Karlin and McGregor [13] we call this model the skewed conditional branching process model.

Schweinsberg [32] studies models, in which $N$ individuals are sampled without replacement from $S_{N}:=X_{1}+\cdots+X_{N}$ offspring, where $X_{1}, \ldots, X_{N}$ are given i.i.d. random variables. The effective population sizes of these models satisfy $N_{e}=$ $O(N)$ (see [32, Eq. (18)]), and, in particular, $N_{e}$ can exhibit a rich variety of sub-linear behavior (see, for example, [32, Lemma 13]), however, these models are different from our models, which are based on conditioning instead of sampling.

The paper is organized as follows. In Section 2 a skewed Cannings model is introduced and analyzed. This model essentially coincides with an associated standard exchangeable Cannings model $[3,4]$. Section 2 therefore heavily gains from the theory on Cannings models. The results of Section 2 are applied in Section 3 to the skewed conditional branching process model. Since this huge class of conditional models has in general a quite complicated probabilistic structure, we further specialize in Section 4 to a subclass of skewed compound Poisson models. Exact formulae for the transition probabilities of the forward and the backward process are derived. It is furthermore shown (Theorem 4.3) that, in the unbiased (nonskewed) case, the model is in the domain of attraction (see Definition 2.1) of the Kingman coalescent. In all cases we obtain exact and asymptotic formulae for the effective population size $N_{e}$, which can deviate substantially from the total population size $N$. The following Sections 5 and 6 are devoted to two particular compound Poisson models, the skewed Wright-Fisher model and the skewed Dirichlet model. In both models the effective population size $N_{e}$ is less than or equal to the total population size $N$, and, depending on the parameter choices, can indeed be substantially smaller than $N$, for example $N_{e} \sim \rho N$ with $\rho \in(0,1), N_{e}=O\left(N^{\beta}\right)$ with $\beta \in(0,1), N_{e}=O(\log N)$, or even $N_{e}=O(1)$. These two models are in particular analyzed for power law skewed fertilities and for geometrically skewed fertilities. It turns out that for a wide range of parameter choices, these models are not anymore in the domain of attraction of the Kingman coalescent. Coalescents allowing for simultaneous multiple collisions arise in the limit as the total population size tends to infinity. The paper finishes in Section 7 with an analog analysis of the skewed Kimura model, a model which does not belong to the compound Poisson class, but nevertheless exhibits similarities with the previously studied models.

Throughout the paper the notation $\mathbb{N}:=\{1,2, \ldots\}, \mathbb{N}_{0}:=\{0,1,2, \ldots\}$, and $[N]:=$ $\{1, \ldots, N\}, N \in \mathbb{N}$, is used. We furthermore use, for fixed $N \in \mathbb{N}$, the symbol $S:=\{0, \ldots, N\}$ for the state space of several discrete processes considered in this paper. Moreover, for $k \in \mathbb{N}_{0}$ and $x \in \mathbb{R},(x)_{k}:=x(x-1) \cdots(x-k+1)$ and 
$[x]_{k}:=x(x+1) \cdots(x+k-1)$ denote the descending and ascending factorials respectively, with the convention that $(x)_{0}=[x]_{0}=1$.

\section{Skewed Cannings models}

Consider a population with constant population size $N \in \mathbb{N}$ evolving in discrete nonoverlapping generations $t \in \mathbb{N}_{0}$. The $n$th individual, $n \in[N]$, of the $t$ th generation gives birth to a random number $\mu_{n}(t)$ of children. Since the population is assumed to have constant population size $N$, for each fixed generation $t \in \mathbb{N}$, the random vector $\mu(t):=\left(\mu_{1}(t), \ldots, \mu_{N}(t)\right)$ must take values in the discrete $N$-simplex $\Delta(N)$ consisting of all $k=\left(k_{1}, \ldots, k_{N}\right) \in \mathbb{N}_{0}^{N}$ satisfying $k_{1}+\cdots+k_{N}=N$. Note that the random variable $\mu_{n}(t)=\mu_{n, N}(t)$ is allowed to depend on the total population size $N$. However, for simplicity, this dependence on $N$ is usually not indicated throughout the paper. It is assumed that the random vectors $\mu(0), \mu(1), \ldots$ are i.i.d., so the model is time-homogeneous. We write $\mu_{n}:=\mu_{n}(0), n \in[N]$, and $\mu:=\mu(0)$ for convenience. Note that the model is in principle defined in the same way as an exchangeable Cannings model $[3,4]$. The only slight modification is that, for each fixed generation $t \in \mathbb{N}_{0}$, the offspring variables $\mu_{1}(t), \ldots, \mu_{N}(t)$ are not necessarily assumed to be exchangeable. Models of this form have been studied in [19] even in the time-inhomogeneous setting when the total population size is not necessarily constant equal to $N$. Particular examples, such as the skewed Wright-Fisher model, which is studied in Section 5 in more detail, have been the source of recurrent interest in the literature (see, for example, [2] and [18]). A main tool exploited in this section is the following shuffling procedure. For each fixed generation $t \in \mathbb{N}_{0}$ let $\nu(t)=\left(\nu_{1}(t), \ldots, \nu_{N}(t)\right)$ be a random permutation (shuffling) of $\mu(t)=\left(\mu_{1}(t), \ldots, \mu_{N}(t)\right)$. Then $\nu(t)$ is exchangeable with distribution

$$
\mathbb{P}(\nu(t)=k)=\frac{1}{N !} \sum_{\pi} \mathbb{P}(\pi \mu(t)=k), \quad k \in \Delta(N),
$$

where $\pi \mu(t):=\left(\mu_{\pi 1}(t), \ldots, \mu_{\pi N}(t)\right)$ and the sum extends over all permutations $\pi$ of $[N]$. We interpret $\nu_{n}(t)$ as the number of offspring of the $n$th individual of the $t$ th generation in an exchangeable Cannings model $[3,4]$. Again we write $\nu_{n}:=\nu_{n}(0)$, $n \in[N]$, and $\nu:=\nu(0)$ for convenience. As we will see soon, this exchangeable Cannings population model will turn out to be very helpful to analyze the original skewed Cannings model. More precisely, since all assignments of offspring to parents are assumed to be equally likely, there is essentially no difference between the skewed model and the associated shuffled exchangeable Cannings model. However, we are interested in these skewed Cannings models since (as the following sections will show) starting with not necessarily exchangeable offspring variables $\mu_{1}, \ldots, \mu_{N}$ naturally leads to interesting subclasses and examples of Cannings models, which to the best of our knowledge have not been introduced so far. Most of the results provided in this section are well known from the literature on Cannings models and from coalescent theory. We chose to present these results, since we interpret them in terms of the original skewed model we started with at the beginning of this section. For example, we clarify how the forward and backward processes of these models depend on the original offspring variables $\mu_{1}, \ldots, \mu_{N}$.

2.1. Looking forwards in time. Take a sample of $n \in S$ individuals of generation 0 and, for $t \in \mathbb{N}_{0}$, let $X_{t}$ denote the number of descendants of these $n$ individuals 
in generation $t$. The process $X:=\left(X_{t}\right)_{t \in \mathbb{N}_{0}}$, called the forward process, is a timehomogeneous Markov chain with state space $S$ and initial state $X_{0}=n$. The transition probabilities $P_{i, j}:=\mathbb{P}\left(X_{t+1}=j \mid X_{t}=i\right), i, j \in S$, must coincide with those of the associated exchangeable Cannings model with offspring distributions (1), since in the considered skewed model all assignments of offspring to parents are assumed to be equally likely. From the literature on exchangeable models (Cannings [3, p. 267]), it follows that $P_{i, j}=\mathbb{P}\left(\nu_{1}+\cdots+\nu_{i}=j\right)=\sum_{k} \mathbb{P}(\nu=k)$, where the sum extends over all $k \in \Delta(N)$ satisfying $k_{1}+\cdots+k_{i}=j$. Plugging in (1) and interchanging the two sums involved yields

$$
P_{i, j}=\frac{1}{N !} \sum_{\pi} \sum_{k} \mathbb{P}(\pi \mu=k)=\frac{1}{N !} \sum_{\pi} \mathbb{P}\left(\mu_{\pi 1}+\cdots+\mu_{\pi i}=j\right) .
$$

Introducing $n_{1}:=\pi 1, \ldots, n_{i}:=\pi i$, and noting that there exist exactly $(N-i)$ ! permutations $\pi$ leaving $\pi 1, \ldots, \pi i$ fixed, it follows that

$$
P_{i, j}=\frac{1}{(N)_{i}} \sum_{\substack{n_{1}, \ldots, n_{i}=1 \\
\text { all distinct }}}^{N} \mathbb{P}\left(\sum_{k=1}^{i} \mu_{n_{k}}=j\right)=\frac{1}{\left(\begin{array}{c}
N \\
i
\end{array}\right)} \sum_{1 \leq n_{1}<\cdots<n_{i} \leq N} \mathbb{P}\left(\sum_{k=1}^{i} \mu_{n_{k}}=j\right)
$$

for $i, j \in S$. One may rewrite (2) as $P_{i, j}=\left(\begin{array}{c}N \\ i\end{array}\right)^{-1} \sum_{M} \mathbb{P}\left(\sum_{m \in M} \mu_{m}=j\right), i, j \in S$, where the sum $\sum_{M}$ extends over all subsets $M$ of $[N]$ satisfying $|M|=i$. The chain $X$ is a martingale, since $\mathbb{E}\left(X_{t+1} \mid X_{t}=i\right)=\sum_{j \in S} j P_{i, j}=\sum_{j \in S} j \mathbb{P}\left(\nu_{1}+\cdots+\nu_{i}=\right.$ $j)=\mathbb{E}\left(\nu_{1}+\cdots+\nu_{i}\right)=i \mathbb{E}\left(\nu_{1}\right)=i, i \in S$. Since $X$ is bounded $\left(0 \leq X_{t} \leq N\right.$ for all $\left.t \in \mathbb{N}_{0}\right), X_{t}$ converges almost surely to some random variable $X_{\infty}$ as $t \rightarrow \infty$, and $\left(X_{t}\right)_{t \in \mathbb{N}_{0} \cup\{\infty\}}$ is still a martingale. If $\mathbb{P}\left(\mu_{k}=1\right)<1$ for some $k \in[N]$, then (see, for example, Section 2.1 of [20]) $X_{\infty}$ takes the two values 0 and $N$ with probability $\mathbb{P}\left(X_{\infty}=0\right)=1-n / N$ and $\mathbb{P}\left(X_{\infty}=N\right)=n / N$ respectively.

The associated Cannings model with exchangeable offspring variables $\nu_{1}, \ldots, \nu_{N}$ is useful in many respects. For instance, in terms of the so-called coalescence probability (see, for example, $[21]$ )

$$
c_{N}:=\frac{\mathbb{E}\left(\left(\nu_{1}\right)_{2}\right)}{N-1}=\frac{\operatorname{Var}\left(\nu_{1}\right)}{N-1}=1-\mathbb{E}\left(\nu_{1} \nu_{2}\right)=-\operatorname{Cov}\left(\nu_{1}, \nu_{2}\right),
$$

the variance of $X_{t+1}$, given $X_{t}=i$, can be expressed as

$$
\begin{aligned}
\operatorname{Var}\left(X_{t+1} \mid X_{t}=i\right) & =\operatorname{Var}\left(\nu_{1}+\cdots+\nu_{i}\right)=i \operatorname{Var}\left(\nu_{1}\right)+i(i-1) \operatorname{Cov}\left(\nu_{1}, \nu_{2}\right) \\
& =i(N-1) c_{N}-i(i-1) c_{N}=i(N-i) c_{N} .
\end{aligned}
$$

Defining the heterogeneity of the population at generation $t$ as $2\left(X_{t} / N\right)\left(1-X_{t} / N\right)$, we have $2 \mathbb{E}\left(\left(X_{t} / N\right)\left(1-X_{t} / N\right) \mid X_{0}=i\right)=2\left(1-c_{N}\right)^{t}(i / N)(1-i / N), t \in \mathbb{N}_{0}, i \in S$, showing that, if $c_{N}>0$, the mean heterogeneity tends to 0 exponentially fast as $t \rightarrow \infty$. We will provide further information on $c_{N}$ when the model is studied backwards in time.

2.2. Looking backwards in time. Instead of looking forwards in time let us now look backwards in time and count, starting with all $N$ individuals from some generation $t_{0} \in \mathbb{N}_{0}$, the number of ancestors of these $N$ individuals $t \in\left\{0, \ldots, t_{0}\right\}$ generations backward in time. More precisely, let $\widehat{X}_{t}$ denote the number of ancestors of the $N$ individuals of generation $t_{0}$ in generation $t_{0}-t$. The process $\widehat{X}:=\left(\widehat{X}_{t}\right)_{t \in\left\{0, \ldots, t_{0}\right\}}$, called the backward process, is a time-homogeneous Markov chain with state space 
$S$ and initial state $N$. The transition probabilities $\widehat{P}_{i, j}:=\mathbb{P}\left(\widehat{X}_{t+1}=j \mid \widehat{X}_{t}=i\right)$, $i, j \in S$, coincide with those of the associated Cannings model with exchangeable offspring vector $\nu$ having distribution (1). Therefore, from the literature on exchangeable models (Cannings [3, Theorem 11], Gladstien [9, Examples]), it follows that

$$
\begin{aligned}
\widehat{P}_{i, j} & =\frac{\left(\begin{array}{c}
N \\
j
\end{array}\right)}{\left(\begin{array}{c}
N \\
i
\end{array}\right)} \sum_{\substack{l_{1}, \ldots, l_{j} \in \mathbb{N} \\
l_{1}+\cdots+l_{j}=i}} \mathbb{E}\left(\prod_{k=1}^{j}\left(\begin{array}{c}
\nu_{k} \\
l_{k}
\end{array}\right)\right) \\
& =\frac{1}{\left(\begin{array}{c}
N \\
i
\end{array}\right)} \sum_{\substack{1 \leq n_{1}<\cdots<n_{j} \leq N \\
l_{1}, \ldots, l_{j} \in \mathbb{N} \\
l_{1}+\cdots+l_{j}=i}} \mathbb{E}\left(\prod_{k=1}^{j}\left(\begin{array}{c}
\mu_{n_{k}} \\
l_{k}
\end{array}\right)\right), \quad i, j \in S,
\end{aligned}
$$

with the convention that $\widehat{P}_{i, 0}=\delta_{i 0}$ (Kronecker symbol). Note that the last equality follows from (1) via a similar argument as already used to derive the formula (2) for $P_{i, j}$. The states 0 and 1 are absorbing.

The matrix $\widehat{P}=\left(\widehat{P}_{i, j}\right)_{i, j \in S}$ is lower left triangular and has hence eigenvalues $\lambda_{i}:=\widehat{P}_{i, i}, i \in S$. From (4) it follows that $\lambda_{0}=1$ and that $\lambda_{i}=\widehat{P}_{i, i}=$ $\left(\begin{array}{c}N \\ i\end{array}\right)^{-1} \sum_{1 \leq n_{1}<\cdots<n_{i} \leq N} \mathbb{E}\left(\mu_{n_{1}} \cdots \mu_{n_{i}}\right), i \in[N]$. Note that $\lambda_{1}=1$ and that $\lambda_{2}=$ $\left(\begin{array}{c}N \\ 2\end{array}\right)^{-1} \sum_{1 \leq n_{1}<n_{2} \leq N} \mathbb{E}\left(\mu_{n_{1}} \mu_{n_{2}}\right)=\mathbb{E}\left(\nu_{1} \nu_{2}\right)=1-c_{N}$. Since the forward and backward transition matrices $P=\left(P_{i, j}\right)_{i, j \in S}$ and $\widehat{P}=\left(\widehat{P}_{i, j}\right)_{i, j \in S}$ coincide with those of the associated Cannings model with exchangeable offspring vector $\nu$, it is allowed to apply results on exchangeable Cannings models, in particular duality results such as the duality relation $P H=H \widehat{P}^{\prime}$, where $H=\left(H_{i j}\right)_{i, j \in S}$ is (see, for example, [20]) the matrix with entries $H_{i j}=\left(\begin{array}{c}i \\ j\end{array}\right) /\left(\begin{array}{c}N \\ j\end{array}\right), i, j \in S$. Since $H$ is non-singular, $P$ has the same eigenvalues as $\widehat{P}$. In particular, $c_{N}=1-\lambda_{2}$ is the spectral gap of $P$ and $\widehat{P}$.

The random variable $\widehat{X}_{t}$ counts the number of ancestors in generation $t_{0}-t$, but it gives no information about whether two individuals $i$ and $j$, randomly picked from generation $t_{0}$, share a common parent in generation $t_{0}-t$. In order to encode this information, a more enriched ancestral process has to be considered, which is now described. Take a random sample of $n \in[N]$ individuals from generation $t_{0} \in \mathbb{N}_{0}$ and, for $t \in\left\{0, \ldots, t_{0}\right\}$, define a random relation $\mathcal{R}_{t}$ on $\{1, \ldots, n\}$ by saying that $(i, j) \in \mathcal{R}_{t}$ if and only if the individuals $i$ and $j$ have a common parent in generation $t_{0}-t$. Note that $\mathcal{R}_{t}={ }_{(N)} \mathcal{R}_{t}^{(n)}$ depends on the sample size $n$ and on the total population size $N$. The process $\left(\mathcal{R}_{t}\right)_{t \in\left\{0, \ldots, t_{0}\right\}}$ is called the ancestral process or a discrete coalescent process. It is well known (see, for example, [19]) that $\left(\mathcal{R}_{t}\right)_{t \in\left\{0, \ldots, t_{0}\right\}}$ is a Markov chain with state space $\mathcal{E}_{n}$, the set of equivalence relations (partitions) on $\{1, \ldots, n\}$, and transition probabilities

$$
\mathbb{P}\left(\mathcal{R}_{t+1}=\eta \mid \mathcal{R}_{t}=\xi\right)=\Phi_{j}\left(l_{1}, \ldots, l_{j}\right), \quad \xi, \eta \in \mathcal{E}_{n}, \xi \subseteq \eta
$$

where

$$
\Phi_{j}\left(l_{1}, \ldots, l_{j}\right):=\frac{1}{(N)_{l_{1}+\cdots+l_{j}}} \sum_{\begin{array}{c}
n_{1}, \ldots, n_{j}=1 \\
\text { all distinct }
\end{array}}^{N} \mathbb{E}\left(\left(\mu_{n_{1}}\right)_{l_{1}} \cdots\left(\mu_{n_{j}}\right)_{l_{j}}\right) .
$$


Here $j:=|\eta|$ denotes the number of equivalence classes (blocks) of $\eta$ and $l_{1}, \ldots, l_{j} \in$ $\mathbb{N}$ are the group sizes of merging classes of $\xi$. Note that $l_{1}+\cdots+l_{j}$ is the number of classes (blocks) of $\xi$. Comparing (6) with (4) shows that

$$
\widehat{P}_{i, j}=\frac{i !}{j !} \sum_{\begin{array}{c}
l_{1}, \ldots, l_{j} \in \mathbb{N} \\
l_{1}+\cdots+l_{j}=i
\end{array}} \frac{\Phi_{j}\left(l_{1}, \ldots, l_{j}\right)}{l_{1} ! \cdots l_{j} !}, \quad i, j \in\{1, \ldots, N\} .
$$

For $j=1,(4)$ and $(7)$ reduce to

$$
\widehat{P}_{i, 1}=\Phi_{1}(i)=\frac{1}{(N)_{i}} \sum_{n=1}^{N} \mathbb{E}\left(\left(\mu_{n}\right)_{i}\right), \quad i \in\{1, \ldots, N\} .
$$

The coalescence probability $c_{N}$, i.e. the probability that two individuals, randomly chosen from some generation, have a common parent, is hence

$$
c_{N}:=\widehat{P}_{2,1}=\Phi_{1}(2)=\frac{1}{(N)_{2}} \sum_{n=1}^{N} \mathbb{E}\left(\left(\mu_{n}\right)_{2}\right),
$$

in agreement with (3), and the effective population size is $N_{e}:=1 / c_{N}$. We will later also make use of the probability that three individuals, randomly chosen from some generation, share a common parent, which is given by

$$
d_{N}:=\widehat{P}_{3,1}=\Phi_{1}(3)=\frac{1}{(N)_{3}} \sum_{n=1}^{N} \mathbb{E}\left(\left(\mu_{n}\right)_{3}\right) .
$$

The transition probabilities (5) do not depend on $t$ and $t_{0}$. It is hence allowed to choose $t_{0}$ arbitrary large. We can therefore think of a process $\left(\mathcal{R}_{t}\right)_{t \in \mathbb{N}_{0}}$ with transition probabilities (5) and time $t \in \mathbb{N}_{0}$. A fundamental result from coalescent theory (see, for example, [21, p. 989)] or [25, Lemma 5.5]) states that, if $d_{N} / c_{N} \rightarrow 0$, then $c_{N} \rightarrow 0$ and, moreover (see [24] or [21, Theorem 4 (b)]), if $d_{N} / c_{N} \rightarrow 0$, then, for each sample size $n \in \mathbb{N}$, the time-scaled process $\left(\mathcal{R}_{\left[t / c_{N}\right]}^{(n)}\right)_{t \in[0, \infty)}$ converges weakly to Kingman's $n$-coalescent $\left(R_{t}^{(n)}\right)_{t \in[0, \infty)}$ as the total population size $N$ tends to infinity. In [24] there is also a criterion in terms of the quantities (6) provided ensuring that, for each $n \in \mathbb{N}$, the time-scaled process $\left(\mathcal{R}_{\left[t / c_{N}\right]}^{(n)}\right)_{t \in[0, \infty)}$ converges weakly as $N \rightarrow \infty$ to a more general process $\left(\varrho_{n} R_{t}\right)_{t \in[0, \infty)}$, where $\varrho_{n}$ denotes the restriction from $\mathcal{E}$, the set of all equivalence relations on $\mathbb{N}$, to $\mathcal{E}_{n}$, and $R=\left(R_{t}\right)_{t \geq 0}$ is a continuous-time coalescent process allowing for simultaneous multiple collisions of ancestral lineages. This asymptotic behavior can only occur if $c_{N} \rightarrow 0$. If, instead, $c_{N}$ converges to a positive constant, then, a similar criterion for the quantities (6), also provided in [24], ensures that, for each sample size $n \in \mathbb{N}$, the process $\left(\mathcal{R}_{t}^{(n)}\right)_{t \in \mathbb{N}_{0}}$ (without any time-scaling involved) converges weakly to a discrete-time process $\left(\varrho_{n} R_{t}\right)_{t \in \mathbb{N}_{0}}$, where $\left(R_{t}\right)_{t \in[0, \infty)}$ is a discrete-time coalescent allowing for simultaneous multiple collisions of ancestral lineages.

Definition 2.1. a) Let $R=\left(R_{t}\right)_{t \in[0, \infty)}$ be a continuous-time coalescent with simultaneous multiple collisions. We say that the considered population model is in the domain of attraction of $R$, if, for each sample size $n \in \mathbb{N}$, the time-scaled ancestral process $\left(\mathcal{R}_{\left[t / c_{N}\right]}^{(n)}\right)_{t \in[0, \infty)}$ converges weakly to $\left(\varrho_{n} R_{t}\right)_{t \in[0, \infty)}$ as $N \rightarrow \infty$.

b) Let $R=\left(R_{t}\right)_{t \in \mathbb{N}_{0}}$ be a discrete-time coalescent with simultaneous multiple collisions. We say that the considered population model is in the domain of attraction 
of $R$, if, for each sample size $n \in \mathbb{N}$, the ancestral process $\left(\mathcal{R}_{t}^{(n)}\right)_{t \in \mathbb{N}_{0}}$ converges weakly to $\left(\varrho_{n} R_{t}\right)_{t \in \mathbb{N}_{0}}$ as $N \rightarrow \infty$.

In both cases we call $R$ the attractor of the considered population model.

Example 2.2. (extended Moran model) Let $U$ be a random variable taking values in $\{0, \ldots, N\}$. Define the offspring vector $\mu=\left(\mu_{1}, \ldots, \mu_{N}\right)$ via $\mu_{n}:=1$ if $n \in\{1, \ldots, N-U\}$, and, on $\{U>0\}, \mu_{n}:=0$ if $n \in\{N-U+1, \ldots, N-1\}$, and $\mu_{N}:=U$. The associated shuffled exchangeable Cannings model was considered by Eldon and Wakeley [6]. For $U \equiv 0$ (and as well for $U \equiv 1$ ), we obtain the trivial model in which every individual has one offspring $\left(\mu_{n}=1\right.$ for all $\left.n \in[N]\right)$. For $U \equiv N$ in this model the $N$ th individual is the parent of all the $N$ children of the next generation $\left(\mu_{N}=N\right)$. If $U \geq 1$ almost surely, then, by (2), the forward chain $X$ has transition probabilities

$$
\begin{aligned}
P_{i, j} & =\frac{1}{\left(\begin{array}{c}
N \\
i
\end{array}\right)}\left(\sum_{1 \leq n_{1}<\cdots<n_{i}<N} \mathbb{P}\left(\sum_{k=1}^{i} \mu_{n_{k}}=j\right)+\sum_{1 \leq n_{1}<\cdots<n_{i}=N} \mathbb{P}\left(\sum_{k=1}^{i-1} \mu_{n_{k}}=j-U\right)\right) \\
& =\frac{1}{\left(\begin{array}{c}
N \\
i
\end{array}\right)} \mathbb{E}\left(\left(\begin{array}{c}
N-U \\
j
\end{array}\right)\left(\begin{array}{c}
U-1 \\
i-j
\end{array}\right)+\left(\begin{array}{c}
N-U \\
j-U
\end{array}\right)\left(\begin{array}{c}
U-1 \\
(i-1)-(j-U)
\end{array}\right)\right) \\
& = \begin{cases}\mathbb{E}\left(\left(\begin{array}{c}
N-U \\
j
\end{array}\right)\left(\begin{array}{c}
U-1 \\
i-j
\end{array}\right)\right) /\left(\begin{array}{c}
N \\
i
\end{array}\right) & \text { if } j<i, \\
\mathbb{E}\left(\left(\begin{array}{c}
N-U \\
j
\end{array}\right)+\left(\begin{array}{c}
N-U \\
N-j
\end{array}\right)\right) /\left(\begin{array}{c}
N \\
i
\end{array}\right)=\mathbb{E}\left(\frac{(i)_{U}+(N-i)_{U}}{(N)_{U}}\right) & \text { if } j=i, \\
\mathbb{E}\left(\left(\begin{array}{c}
N-U \\
N-j
\end{array}\right)\left(\begin{array}{c}
U-1 \\
j-i
\end{array}\right)\right) /\left(\begin{array}{c}
N \\
i
\end{array}\right) & \text { if } j>i .\end{cases}
\end{aligned}
$$

If $\mathbb{P}(U=0)>0$, then in the above expressions for $P_{i, j}$ each $U$ has to be replaced by $\max (U, 1)$. For $U \equiv 2$ this model reduces to the standard Moran model with forward transition probabilities $P_{i, i-1}=i(N-i) /(N)_{2}, i \in\{1, \ldots, N\}, P_{i, i+1}=$ $i(N-i) /(N)_{2}, i \in\{0, \ldots, N-1\}, P_{i, i}=1-2 i(N-i) /(N)_{2}, i \in\{0, \ldots, N\}$, and $P_{i, j}=0$ otherwise. From (4), it follows similarly that, for $i, j \in[N]$,

$$
\widehat{P}_{i, j}= \begin{cases}\mathbb{E}\left(\left(\begin{array}{c}
N-U \\
j-1
\end{array}\right)\left(\begin{array}{c}
U \\
i-j+1
\end{array}\right)\right) /\left(\begin{array}{c}
N \\
i
\end{array}\right) & \text { if } j<i \\
\mathbb{E}\left(\left(\begin{array}{c}
N-U \\
i
\end{array}\right)+U\left(\begin{array}{c}
N-U \\
i-1
\end{array}\right)\right) /\left(\begin{array}{c}
N \\
i
\end{array}\right) & \text { if } j=i \\
0 & \text { if } j>i\end{cases}
$$

Note that $\widehat{P}_{i, 1}=\mathbb{E}\left((U)_{i}\right) /(N)_{i}, i \in\{2, \ldots, N\}$. In particular, $c_{N}=\widehat{P}_{2,1}=$ $\mathbb{E}\left((U)_{2}\right) /(N)_{2}$, in agreement with $\left[6\right.$, Eq. (2)], and $d_{N}=\widehat{P}_{3,1}=\mathbb{E}\left((U)_{3}\right) /(N)_{3}$. The model is in the domain of attraction of the Kingman coalescent if and only if $\mathbb{E}\left((U)_{3}\right) /\left(N \mathbb{E}\left((U)_{2}\right)\right) \rightarrow 0$ as $N \rightarrow \infty$. If $U$ is binomially distributed with parameters $N$ and $p \in[0,1]$, then $c_{N}=p^{2}$. Note that $p=p_{N}$ may depend on $N$, so this model can have a wide variety of effective population sizes $N_{e}=1 / p_{N}^{2}$. For instance, if $p_{N}=N^{-\alpha}, \alpha>0$, then $N_{e}=N^{2 \alpha}$ is sub-linear for $\alpha<1 / 2$ and super-linear for $\alpha>1 / 2$. If $p_{N}=\lambda^{N}, \lambda<1$, then $N_{e}=\lambda^{-2 N}$ grows exponentially. We will come back to this model in Proposition 3.1.

In the following section we will introduce a skewed conditional branching process model, which can be viewed as a particular Cannings model. We will later identify the attractor $R$ of several concrete such population models. 


\section{Skewed CONDItional BRANCHING PROCESS MODELS}

Let $\xi_{1}, \xi_{2}, \ldots$ be independent non-negative integer valued random variables and let $f_{n}$ denote the probability generating function (pgf) of $\xi_{n}, n \in \mathbb{N}$. For any pgf $g$ and $l \in \mathbb{N}_{0}$ we use the standard notation $g^{l}$ for the $l$ th power of $g\left(g^{0}=1\right)$ and the notation $g^{(l)}$ for the $l$ th derivative of $g$. Moreover, $\left[x^{l}\right] g(x)$ denotes the coefficient in front of $x^{l}$ in the Taylor expansion of $g$ around zero. For $N \in \mathbb{N}$ assume that $\mathbb{P}\left(\xi_{1}+\cdots+\xi_{N}=N\right)>0$ and let $\mu=\left(\mu_{1}, \ldots, \mu_{N}\right)$ be a random vector with distribution

$$
\mathbb{P}(\mu=k):=\frac{\mathbb{P}\left(\xi_{1}=k_{1}\right) \cdots P\left(\xi_{N}=k_{N}\right)}{\mathbb{P}\left(\xi_{1}+\cdots+\xi_{N}=N\right)}=\frac{\left[x^{k_{1}}\right] f_{1}(x) \cdots\left[x^{k_{N}}\right] f_{N}(x)}{\left[x^{N}\right]\left(f_{1} \cdots f_{N}\right)(x)},
$$

$k=\left(k_{1}, \ldots, k_{N}\right) \in \Delta(N)$. The distribution of $\mu$ is hence that of $\left(\xi_{1}, \ldots, \xi_{N}\right)$ conditioned on the event that $\xi_{1}+\cdots+\xi_{N}=N$. Note that, for $n \in[N]$, the marginal variable $\mu_{n}$ has distribution

$$
\begin{aligned}
\mathbb{P}\left(\mu_{n}=k\right) & =\frac{\mathbb{P}\left(\xi_{n}=k\right) \mathbb{P}\left(\sum_{m \in[N] \backslash\{n\}} \xi_{m}=N-k\right)}{\mathbb{P}\left(\xi_{1}+\cdots+\xi_{N}=N\right)} \\
& =\frac{\left(\left[x^{k}\right] f_{n}(x)\right)\left(\left[x^{N-k}\right]\left(\prod_{m \in[N] \backslash\{n\}} f_{m}(x)\right)\right)}{\left[x^{N}\right]\left(f_{1} \cdots f_{N}\right)(x)}, \quad k \in\{0, \ldots, N\} .
\end{aligned}
$$

For each $n \in[N]$ one may interpret $\mu_{n}$ as the number of offspring of individual $n$ in a population with non-overlapping generations of constant population size $N$. Note that $\mu$ has pgf

$$
\mathbb{E}\left(z_{1}^{\mu_{1}} \cdots z_{N}^{\mu_{N}}\right)=\frac{\left[x^{N}\right]\left(f_{1}\left(x z_{1}\right) \cdots f_{N}\left(x z_{N}\right)\right)}{\left[x^{N}\right]\left(f_{1} \cdots f_{N}\right)(x)}, \quad\left|z_{1}\right|, \ldots,\left|z_{N}\right| \leq 1 .
$$

Let $l_{1}, \ldots, l_{N} \in \mathbb{N}_{0}$ and put $l:=l_{1}+\cdots+l_{N}$. Applying the 'derivative operator' $\left(\partial^{l_{1}} / \partial^{l_{1}} z_{1}\right) \cdots\left(\partial^{l_{N}} / \partial^{l_{N}} z_{N}\right)$ to the left-hand side and the right-hand side of $(9)$, and noting that it is allowed to interchange this derivative operator with the 'coefficient operator' $\left[x^{N}\right]$ in the numerator on the right-hand side of $(9)$, it follows that

$$
\mathbb{E}\left(\left(\mu_{1}\right)_{l_{1}} z_{1}^{\mu_{1}-l_{1}} \cdots\left(\mu_{N}\right)_{l_{N}} z_{N}^{\mu_{N}-l_{N}}\right)=\frac{\left[x^{N-l}\right]\left(\prod_{i=1}^{N} f_{i}^{\left(l_{i}\right)}\left(x z_{i}\right)\right)}{\left[x^{N}\right]\left(f_{1} \cdots f_{N}\right)(x)} .
$$

Taking the limit $z_{1} \nearrow 1, \ldots, z_{N} \nearrow 1$ shows that $\mu$ has descending factorial moments

$$
\mathbb{E}\left(\left(\mu_{1}\right)_{l_{1}} \cdots\left(\mu_{N}\right)_{l_{N}}\right)=\frac{\left[x^{N-l}\right]\left(\prod_{i=1}^{N} f_{i}^{\left(l_{i}\right)}(x)\right)}{\left[x^{N}\right]\left(f_{1} \cdots f_{N}\right)(x)}, \quad l_{1}, \ldots, l_{N} \in \mathbb{N}_{0} .
$$

If the random variables $\xi_{1}, \xi_{2}, \ldots$ are identically distributed, then the model reduces to the conditional branching process model first introduced by Moran and Watterson [26] and further investigated for example by Karlin and McGregor [13]. In this case, for each fixed $N \in \mathbb{N}$, the random variables $\mu_{1}, \ldots, \mu_{N}$ are exchangeable. Note however, that in general it is not assumed here that the random variables $\xi_{1}, \xi_{2}, \ldots$ are identically distributed. In this sense the model is a bit more general than the conditional branching process model of [26]. Particular classes and examples of the model, which to the best of our knowledge are new or only briefly mentioned in the literature, are introduced and analyzed in the following sections, among them the skewed Wright-Fisher model (Section 5), the skewed Dirichlet model (Section 6 ), and the skewed Kimura model (Section 7). One may think that, at least for fixed $N$, the model can be reduced to a simpler model by randomly permutating 
the random variables $\xi_{1}, \ldots, \xi_{N}$. However, this is not the case. If $\eta_{1}, \ldots, \eta_{N}$ denotes a random permutation of $\xi_{1}, \ldots, \xi_{N}$, then the random variables $\eta_{1}, \ldots, \eta_{N}$ are identically distributed but in general not independent (not even uncorrelated) anymore. In this sense the model is indeed more general than the conditional branching process model of Karlin and McGregor.

On the other hand, for arbitrary but fixed $N \in \mathbb{N}$, the model is well known from the literature in the following sense. Fix $N \in \mathbb{N}$ and let $\nu=\left(\nu_{1}, \ldots, \nu_{N}\right)$ be a random permutation of $\mu=\left(\mu_{1}, \ldots, \mu_{N}\right)$. As explained in Section 2, the model can be interpreted as an exchangeable Cannings model with population size $N$ and offspring vector $\nu$. Note that, for $k=\left(k_{1}, \ldots, k_{N}\right) \in \Delta(N)$,

$$
\begin{aligned}
\frac{\mathbb{P}\left(\eta_{1}=k_{1}, \ldots, \eta_{N}=k_{N}\right)}{\mathbb{P}\left(\eta_{1}+\cdots+\eta_{N}=N\right)} & =\frac{1}{N !} \sum_{\pi} \frac{\mathbb{P}\left(\xi_{\pi 1}=k_{1}, \ldots, \xi_{\pi N}=k_{N}\right)}{\mathbb{P}\left(\xi_{1}+\cdots+\xi_{N}=N\right)} \\
& =\frac{1}{N !} \sum_{\pi} \mathbb{P}(\pi \mu=k)=\mathbb{P}(\nu=k),
\end{aligned}
$$

so permutating the random variables $\xi_{1}, \ldots, \xi_{N}$ (and conditioning) leads to the same exchangeable Cannings model as permutating the random variables $\mu_{1}, \ldots, \mu_{N}$. Essentially all results known for exchangeable Cannings models apply to our model, which simplifies our further analysis significantly. For example, as explained in Section 2, the transition matrix of the forward process has entries (2). Noting that, for any subset $M$ of $[N]$,

$$
\begin{aligned}
\mathbb{P}\left(\sum_{m \in M} \mu_{m}=j\right) & =\frac{\mathbb{P}\left(\sum_{m \in M} \xi_{m}=j\right) \mathbb{P}\left(\sum_{m \in[N] \backslash M} \xi_{m}=N-j\right)}{\mathbb{P}\left(\xi_{1}+\cdots+\xi_{N}=N\right)} \\
& =\frac{\left(\left[x^{j}\right]\left(\prod_{m \in M} f_{m}\right)(x)\right)\left(\left[x^{N-j}\right]\left(\prod_{m \in[N] \backslash M} f_{m}\right)(x)\right)}{\left[x^{N}\right]\left(f_{1} \cdots f_{N}\right)(x)},
\end{aligned}
$$

it follows that the forward transition matrix $P=\left(P_{i, j}\right)_{i, j \in S}$ has entries

$$
P_{i, j}=\frac{1}{\left(\begin{array}{c}
N \\
i
\end{array}\right)} \sum_{M} \frac{\left(\left[x^{j}\right]\left(\prod_{m \in M} f_{m}\right)(x)\right)\left(\left[x^{N-j}\right]\left(\prod_{m \in[N] \backslash M} f_{m}\right)(x)\right)}{\left[x^{N}\right]\left(f_{1} \cdots f_{N}\right)(x)}, \quad i, j \in S,
$$

where the sum $\sum_{M}$ extends over all subsets $M$ of $[N]$ satisfying $|M|=i$. We now turn to the backward chain. From (4) and (10), it follows that the backward transition probabilities are of the form

$$
\begin{aligned}
\widehat{P}_{i, j}= & \frac{1}{\left(\begin{array}{c}
N \\
i
\end{array}\right)} \sum_{1 \leq n_{1}<\cdots<n_{j} \leq N} \\
(12) \quad & \sum_{\substack{l_{1}, \ldots, l_{j} \in \mathbb{N} \\
l_{1}+\cdots+l_{j}=i}} \frac{\left[x^{N-i}\right]\left(f_{n_{1}}^{\left(l_{1}\right)}(x) \cdots f_{n_{j}}^{\left(l_{j}\right)}(x) \prod_{m \in[N] \backslash\left\{n_{1}, \ldots, n_{j}\right\}} f_{m}(x)\right)}{l_{1} ! \cdots l_{j} !\left[x^{N}\right]\left(f_{1} \cdots f_{N}\right)(x)}, \quad i, j \in S,
\end{aligned}
$$

with the convention that $\widehat{P}_{i, 0}=\delta_{i 0}, i \in S$. In particular,

$$
\widehat{P}_{i, 1}=\Phi_{1}(i)=\frac{1}{(N)_{i}} \sum_{n=1}^{N} \frac{\left[x^{N-i}\right]\left(f_{n}^{(i)}(x) \prod_{m \in[N] \backslash\{n\}} f_{m}(x)\right)}{\left[x^{N}\right]\left(f_{1} \cdots f_{N}\right)(x)}, \quad i \in S,
$$


and the coalescence probability is given by

$$
c_{N}=\widehat{P}_{2,1}=\frac{1}{(N)_{2}} \sum_{n=1}^{N} \frac{\left[x^{N-2}\right]\left(f_{n}^{\prime \prime}(x) \prod_{m \in[N] \backslash\{n\}} f_{m}(x)\right)}{\left[x^{N}\right]\left(f_{1} \cdots f_{N}\right)(x)} .
$$

Moreover, the eigenvalues $\lambda_{i}:=\widehat{P}_{i, i}=\Phi_{i}(1, \ldots, 1), i \in S$, of the matrix $\widehat{P}$ are given by $\lambda_{0}=1$ and

$$
\lambda_{i}=\frac{1}{\left(\begin{array}{c}
N \\
i
\end{array}\right)} \sum_{1 \leq n_{1}<\cdots<n_{i} \leq N} \frac{\left[x^{N-i}\right]\left(f_{n_{1}}^{\prime}(x) \cdots f_{n_{i}}^{\prime}(x) \prod_{m \in[N] \backslash\left\{n_{1}, \ldots, n_{i}\right\}} f_{m}(x)\right)}{\left[x^{N}\right]\left(f_{1} \cdots f_{N}\right)(x)}
$$

for $i \in\{1, \ldots, N\}$.

Remark. One may write $\widehat{P}_{i, j}$ in the form

$$
\begin{aligned}
\widehat{P}_{i, j}=\frac{1}{\left(\begin{array}{c}
N \\
i
\end{array}\right)} & \frac{1}{\left[x^{N}\right]\left(f_{1} \cdots f_{N}\right)(x)} \\
& \sum_{1 \leq n_{1}<\cdots<n_{j} \leq N}\left[x^{N-i}\right]\left(S_{f_{n_{1}}, \ldots, f_{n_{j}}, x}(i, j) \prod_{m \in[N] \backslash\left\{n_{1}, \ldots, n_{j}\right\}} f_{m}(x)\right),
\end{aligned}
$$

where (see, for example, [23, Eq. (18)])

$$
S_{g_{1}, \ldots, g_{j}, x}(i, j):=\frac{i !}{j !} \sum_{\substack{l_{1}, \ldots, l_{j} \in \mathbb{N} \\ l_{1}+\cdots+l_{j}=i}} \frac{g_{1}^{\left(l_{1}\right)}(x)}{l_{1} !} \cdots \frac{g_{j}^{\left(l_{j}\right)}(x)}{l_{j} !}
$$

are some sort of generalized Stirling numbers of the second kind, with the convention that, for $j=0, S_{g_{1}, \ldots, g_{j}, x}(i, j)=\delta_{i 0}, i \in \mathbb{N}_{0}$.

Remark. The class of the conditional branching process models leads to a wide variety of Cannings models. However (see the following proposition), not all Cannings models are conditional branching process models.

Proposition 3.1. The extended Moran model (see Example 2.2) is not a conditional branching process model, provided that $\mathbb{P}(2 \leq U \leq N-1)=1$.

Proof. Suppose that the extended Moran model with population size $N$ is a conditional branching process model. Then, there exist i.i.d. random variables $\xi_{1}, \xi_{2}, \ldots$ such that $\mathbb{P}\left(\xi_{1}+\cdots+\xi_{N}=N\right) \neq 0$ and $\mathbb{P}(\nu=k)=p_{k_{1}} \cdots p_{k_{N}} / \mathbb{P}\left(\xi_{1}+\cdots+\xi_{N}=N\right)$ for $k=\left(k_{1}, \ldots, k_{N}\right) \in \Delta(N)$, where $p_{k}:=\mathbb{P}\left(\xi_{1}=k\right), k \in \mathbb{N}_{0}$. In particular,

$$
\begin{aligned}
0 & \neq \mathbb{E}\left(\frac{1}{N\left(\begin{array}{l}
N-1 \\
U-1
\end{array}\right)}\right) \\
& =\mathbb{P}\left(\nu_{1}=\cdots=\nu_{N-U}=1, \nu_{N-U+1}=\cdots=\nu_{N-1}=0, \nu_{N}=U\right) \\
& =\frac{\mathbb{E}\left(p_{0}^{U-1} p_{1}^{N-U} p_{U}\right)}{\mathbb{P}\left(\xi_{1}+\cdots+\xi_{N}=N\right)},
\end{aligned}
$$

and, hence, $p_{1} \neq 0$, since $\mathbb{P}(N-U \geq 1)=1$ by assumption. On the other hand,

$0=\mathbb{P}\left(\nu_{1}=\cdots=\nu_{N}=1\right)=\frac{\mathbb{P}\left(\xi_{1}=1\right) \cdots \mathbb{P}\left(\xi_{N}=1\right)}{\mathbb{P}\left(\xi_{1}+\cdots+\xi_{N}=N\right)}=\frac{p_{1}^{N}}{\mathbb{P}\left(\xi_{1}+\cdots+\xi_{N}=N\right)}$,

and, hence, $p_{1}=0$, an obvious contradiction. In particular, for $N \geq 3$, the standard Moran model $(U \equiv 2)$ is not a conditional branching process model. 
It seems to be hard to derive further exact or asymptotic results as $N \rightarrow \infty$ for the general skewed conditional branching process model. We therefore focus in the following sections on important subclasses.

\section{The SKewed COMPound Poisson Class}

Let $\phi$ be a given power series of the form $\phi(z)=\sum_{m=1}^{\infty} \phi_{m} z^{m} / m !,|z|<r$ with positive radius $r \in(0, \infty]$ of convergence and with non-negative coefficients $\phi_{m} \geq 0$, $m \in \mathbb{N}$. We also assume that $\phi_{1}>0$. Let furthermore $\theta_{1}, \theta_{2}, \ldots \in(0, \infty)$ be given strictly positive real parameters. In this section it is assumed that, for each $n \in \mathbb{N}$, the random variable $\xi_{n}$ of the skewed conditional branching process model (as described in the previous section) has pgf

$$
f_{n}(x)=\mathbb{E}\left(x^{\xi_{n}}\right)=\exp \left(-\theta_{n} \phi(z)\left(1-\frac{\phi(z x)}{\phi(z)}\right)\right), \quad|x| \leq 1 .
$$

In (13), $z$ is viewed as a fixed parameter. However, for the following approach we also see $z$ as a variable satisfying $|z|<r$. In order to state the following lemma we need to introduce, for $\theta>0$, the Taylor expansion

$$
\exp (\theta \phi(z))=\sum_{k=0}^{\infty} \frac{\sigma_{k}(\theta)}{k !} z^{k}, \quad|z|<r,
$$

of $\exp (\theta \phi(z))$, seen as a function of $z$. Note that the coefficients $\sigma_{k}(\theta)$ are strictly positive and they depend on the sequence $\phi .:=\left(\phi_{n}\right)_{n \in \mathbb{N}}$. More precisely, the coefficients $\sigma_{k}(\theta)$ satisfy the recursion $\sigma_{0}(\theta)=1$ and

$$
\sigma_{k+1}(\theta)=\theta \sum_{l=0}^{k}\left(\begin{array}{l}
k \\
l
\end{array}\right) \phi_{k-l+1} \sigma_{l}(\theta), \quad k \in \mathbb{N}_{0},
$$

i.e. $\sigma_{1}(\theta)=\theta \phi_{1}, \sigma_{2}(\theta)=\theta \phi_{2}+\theta^{2} \phi_{1}^{2}, \sigma_{3}(\theta)=\theta \phi_{3}+3 \theta^{2} \phi_{1} \phi_{2}+\theta^{3} \phi_{1}^{3}$, and so on. Note that, for each fixed $k \in \mathbb{N}, \sigma_{k}(\theta) \sim\left(\theta \phi_{1}\right)^{k}$ as $\theta \rightarrow \infty$.

Proposition 4.1. If, for each $n \in \mathbb{N}$, the random variable $\xi_{n}$ has a pgf of the form (13), then the forward process $X$ of the associated skewed conditional branching process model has transition probabilities

$$
P_{i, j}=\frac{\left(\begin{array}{c}
N \\
j
\end{array}\right)}{\left(\begin{array}{c}
N \\
i
\end{array}\right)} \sum_{M} \frac{\sigma_{j}\left(\sum_{m \in M} \theta_{m}\right) \sigma_{N-j}\left(\sum_{m \in[N] \backslash M} \theta_{m}\right)}{\sigma_{N}\left(\Theta_{N}\right)}, \quad i, j \in S,
$$

where $\Theta_{N}:=\theta_{1}+\cdots+\theta_{N}$, the sum $\sum_{M}$ extends over all subsets $M \subseteq[N]$ satisfying $|M|=i$, and the coefficients $\sigma_{k}(\theta)$ are recursively defined via (15).

Proof. For $j \in[N], \theta>0$ and $|x| \leq 1$, it follows from (14) that

$$
\left[x^{j}\right] \exp (\theta \phi(z x))=\left[x^{j}\right] \sum_{k=0}^{\infty} \frac{\sigma_{k}(\theta)}{k !}(z x)^{k}=z^{j} \frac{\sigma_{j}(\theta)}{j !} .
$$

Using, for $M \subseteq[N]$, the shortage $\theta:=\sum_{m \in M} \theta_{m}$, it follows that

$$
\begin{aligned}
{\left[x^{j}\right]\left(\sum_{m \in M} f_{m}\right)(x) } & =\left[x^{j}\right] \exp \left(-\theta \phi(z)\left(1-\frac{\phi(z x)}{\phi(z)}\right)\right) \\
& =\exp (-\theta \phi(z))\left[x^{j}\right] \exp (\theta \phi(z x))=\exp (-\theta \phi(z)) z^{j} \frac{\sigma_{j}(\theta)}{j !} .
\end{aligned}
$$


Thus, (16) follows from (11).

Remark. For the unbiased case, when the parameter $\theta_{n}=\theta$ does not depend on $n \in \mathbb{N}$, (16) reduces to

$$
P_{i, j}=\left(\begin{array}{c}
N \\
j
\end{array}\right) \frac{\sigma_{j}(i \theta) \sigma_{N-j}((N-i) \theta)}{\sigma_{N}(N \theta)}, \quad i, j \in S
$$

Let us now turn to the backward process.

Proposition 4.2. If, for each $n \in \mathbb{N}$, the random variable $\xi_{n}$ has a pgf of the form (13), then the backward process $\widehat{X}$ of the associated skewed conditional branching process model has transition probabilities

$$
\widehat{P}_{i, j}=\frac{i !}{\sigma_{i}\left(\Theta_{N}\right)} \sum_{1 \leq n_{1}<\cdots<n_{j} \leq N} \sum_{\substack{l_{1}, \ldots, l_{j} \in \mathbb{N} \\ l_{1}+\cdots+l_{j}=i}} \frac{\sigma_{l_{1}}\left(\theta_{n_{1}}\right) \cdots \sigma_{l_{j}}\left(\theta_{n_{j}}\right)}{l_{1} ! \cdots l_{j} !}, \quad i, j \in S
$$

with the convention that $\widehat{P}_{i, 0}=\delta_{i 0}, i \in S$. Here $\Theta_{N}:=\theta_{1}+\cdots+\theta_{N}$ and the coefficients $\sigma_{k}(\theta)$ are recursively defined via (15). In particular,

$$
\widehat{P}_{i, 1}=\frac{1}{\sigma_{i}\left(\Theta_{N}\right)} \sum_{n=1}^{N} \sigma_{i}\left(\theta_{n}\right), \quad i \in\{1, \ldots, N\}
$$

Proof. From Kolchin's representation formula [15] (see also [28, Chapter 1, Theorem 1.2]), it follows that $\mu=\left(\mu_{1}, \ldots, \mu_{N}\right)$ has distribution

$$
\mathbb{P}(\mu=k)=\frac{N !}{\sigma_{N}\left(\Theta_{N}\right)} \prod_{n=1}^{N} \frac{\sigma_{k_{n}}\left(\theta_{n}\right)}{k_{n} !}, \quad k=\left(k_{1}, \ldots, k_{N}\right) \in \Delta(N) .
$$

Therefore, $\mu$ has joint descending factorial moments

$$
\left.\mathbb{E}\left(\left(\mu_{1}\right)_{l_{1}}\right) \cdots\left(\mu_{N}\right)_{l_{N}}\right)=\frac{(N)_{l_{1}+\cdots+l_{N}}}{\sigma_{l_{1}+\cdots+l_{N}}\left(\Theta_{N}\right)} \prod_{n=1}^{N} \sigma_{l_{n}}\left(\theta_{n}\right), \quad l_{1}, \ldots, l_{N} \in \mathbb{N}_{0}
$$

The probability (6) is therefore of the form

$$
\begin{aligned}
\Phi_{j}\left(l_{1}, \ldots, l_{j}\right) & =\frac{1}{(N)_{l_{1}+\cdots+l_{j}}} \sum_{\substack{n_{1}, \ldots, n_{j}=1 \\
\text { all distinct }}}^{N} \mathbb{E}\left(\left(\mu_{n_{1}}\right)_{l_{1}} \cdots\left(\mu_{n_{j}}\right)_{l_{j}}\right) \\
& =\frac{1}{\sigma_{l_{1}+\cdots+l_{j}}\left(\Theta_{N}\right)} \sum_{\substack{n_{1}, \ldots, n_{j}=1 \\
\text { all distinct }}}^{N} \sigma_{l_{1}}\left(\theta_{n_{1}}\right) \cdots \sigma_{l_{j}}\left(\theta_{n_{j}}\right), \quad l_{1}, \ldots, l_{j} \in \mathbb{N} .
\end{aligned}
$$


Using (7), it follows that

$$
\begin{aligned}
& \widehat{P}_{i, j}=\frac{i !}{j !} \sum_{\substack{l_{1}, \ldots, l_{j} \in \mathbb{N} \\
l_{1}+\cdots+l_{j}=i}} \frac{\Phi_{j}\left(l_{1}, \ldots, l_{j}\right)}{l_{1} ! \cdots l_{j} !} \\
& =\frac{i !}{j ! \sigma_{i}\left(\Theta_{N}\right)} \sum_{\substack{n_{1}, \ldots, n_{j}=1 \\
\text { all distinct }}}^{N} \sum_{\substack{l_{1}, \ldots, l_{j} \in \mathbb{N} \\
l_{1}+\cdots+l_{j}=i}} \frac{\sigma_{l_{1}}\left(\theta_{n_{1}}\right) \cdots \sigma_{l_{j}}\left(\theta_{n_{j}}\right)}{l_{1} ! \cdots l_{j} !} \\
& =\frac{i !}{\sigma_{i}\left(\Theta_{N}\right)} \sum_{1 \leq n_{1}<\cdots<n_{j} \leq N} \sum_{\substack{l_{1}, \ldots, l_{j} \in \mathbb{N} \\
l_{1}+\cdots+l_{j}=i}} \frac{\sigma_{l_{1}}\left(\theta_{n_{1}}\right) \cdots \sigma_{l_{j}}\left(\theta_{n_{j}}\right)}{l_{1} ! \cdots l_{j} !} .
\end{aligned}
$$

Remark. The previous proposition in particular shows that

$$
c_{N}=\widehat{P}_{2,1}=\frac{1}{\sigma_{2}\left(\Theta_{N}\right)} \sum_{n=1}^{N} \sigma_{2}\left(\theta_{n}\right)=\frac{\phi_{2} \Theta_{N}+\phi_{1}^{2} \sum_{n=1}^{N} \theta_{n}^{2}}{\phi_{2} \Theta_{N}+\phi_{1}^{2} \Theta_{N}^{2}} .
$$

Since $N \sum_{n=1}^{N} \theta_{n}^{2} \geq \Theta_{N}^{2}$, it follows that $c_{N} \geq 1 / N$, or, equivalently, that $N_{e} \leq N$.

Let us now focus on the unbiased case, when all the parameters $\theta_{n}=\theta$ are equal to some constant $\theta \in(0, \infty)$. It turns out to be convenient to introduce, for any formal series $a(z)=\sum_{k=1}^{\infty} a_{k} z^{k} / k$ ! or, equivalently, for any sequence $a .:=\left(a_{1}, a_{2}, \ldots\right)$, the Bell polynomials

$$
B_{i, j}(a .):=\frac{i !}{j !}\left[z^{i}\right](a(z))^{j}=\frac{i !}{j !} \sum_{\substack{l_{1}, \ldots, l_{j} \in \mathbb{N} \\ l_{1}+\cdots+l_{j}=i}} \frac{a_{l_{1}} \cdots a_{l_{j}}}{l_{1} ! \cdots l_{j} !}, \quad i, j \in \mathbb{N}_{0} .
$$

Note that $B_{0,0}\left(a_{\text {. }}\right)=1, B_{i, 0}\left(a_{\text {. }}\right)=B_{0, i}\left(a_{\text {. }}\right)=0, B_{i, 1}\left(a_{\text {. }}\right)=a_{i}$ and $B_{i, i}\left(a_{\text {. }}\right)=a_{1}^{i}$ for $i \in \mathbb{N}$. For more information on these polynomials we refer the reader to [1] and Chapter 1 of Pitman [28].

The following theorem provides exact and asymptotic formulae for the transition probabilities $\widehat{P}_{i, j}$ and clarifies that the unbiased compound Poisson class is in the domain of attraction of the Kingman coalescent.

Theorem 4.3. If $\theta_{n}=\theta \in(0, \infty)$ for all $n \in \mathbb{N}$, then

$$
\widehat{P}_{i, j}=\frac{(N)_{j}}{\sigma_{i}(\theta N)} B_{i, j}(\sigma .(\theta)) \quad i, j \in S,
$$

where the $B_{i, j}(\sigma .(\theta))$ are the Bell polynomials of $\sigma .(\theta):=\left(\sigma_{1}(\theta), \sigma_{2}(\theta), \ldots\right)$. In particular,

$$
\widehat{P}_{i, 1}=\frac{N \sigma_{i}(\theta)}{\sigma_{i}(\theta N)}, \quad i \in\{1, \ldots, N\} .
$$

Moreover, for $i, j \in \mathbb{N}$ with $i \geq j$,

$$
\widehat{P}_{i, j} \sim \frac{B_{i, j}(\sigma .(\theta))}{\left(\theta \phi_{1}\right)^{i}} \frac{1}{N^{i-j}}, \quad N \rightarrow \infty,
$$

and the model is in the domain of attraction of the Kingman coalescent in the sense of Definition 2.1 a). 
Proof. Since $\theta_{n}=\theta$ does not depend on $n$, the formula (17) reduces to (19) thanks to the formula (18) for the Bell polynomials. For $j=1,(19)$ reduces to (20), since $B_{i, 1}(\sigma .(\theta))=\sigma_{i}(\theta)$. For $i, j \in \mathbb{N}$ with $i \geq j$ and for all $N \geq i$ it follows that

$$
\widehat{P}_{i, j}=\frac{(N)_{j}}{\sigma_{i}(\theta N)} B_{i, j}(\sigma .(\theta)) \sim \frac{N^{j}}{\left(\theta N \phi_{1}\right)^{i}} B_{i, j}\left(\sigma_{.}(\theta)\right)=\frac{B_{i, j}\left(\sigma_{.}(\theta)\right)}{\left(\theta \phi_{1}\right)^{i}} \frac{1}{N^{i-j}},
$$

which is (21). For $j=1$, Eq. (21) reduces to $\widehat{P}_{i, 1} \sim \sigma_{i}(\theta) /\left(\left(\theta \phi_{1}\right)^{i} N^{i-1}\right)$ as $N \rightarrow \infty$. Thus, $d_{N} / c_{N}=\widehat{P}_{3,1} / \widehat{P}_{2,1} \sim\left(\sigma_{3}(\theta) /\left(\theta \phi_{1} \sigma_{2}(\theta)\right) N^{-1} \rightarrow 0\right.$, which ensures that the model is in the domain of attraction of the Kingman coalescent.

Remark. Theorem 4.3 in particular provides explicit exact formulae for the coalescence probability $c_{N}=\widehat{P}_{2,1}$ and for $d_{N}=\widehat{P}_{3,1}$, namely

$$
c_{N}=\frac{N \sigma_{2}(\theta)}{\sigma_{2}(\theta N)}=\frac{N\left(\theta \phi_{2}+\theta^{2} \phi_{1}^{2}\right)}{\theta N \phi_{2}+(\theta N)^{2} \phi_{1}^{2}}
$$

and

$$
d_{N}=\frac{N \sigma_{3}(\theta)}{\sigma_{3}(\theta N)}=\frac{N\left(\theta \phi_{3}+3 \theta^{2} \phi_{1} \phi_{2}+\theta^{3} \phi_{1}^{3}\right)}{\theta N \phi_{3}+3(\theta N)^{2} \phi_{1} \phi_{2}+(\theta N)^{3} \phi_{1}^{3}} .
$$

Example 4.4. (Wright-Fisher model) For the Wright-Fisher model, $\phi(z)=z$, $\sigma_{k}(\theta)=\theta^{k}$. From $B_{i, j}\left(\theta, \theta^{2}, \ldots\right)=\theta^{i} B_{i, j}(1,1, \ldots)=\theta^{i} S(i, j)$, where the $S(i, j)$ are the Stirling numbers of the second kind, it follows that

$$
\widehat{P}_{i, j}=\frac{(N)_{j}}{\sigma_{i}(\theta N)} B_{i, j}(\sigma .(\theta))=\frac{(N)_{j}}{(\theta N)^{i}} \theta^{i} S(i, j)=(N){ }_{j} N^{-i} S(i, j),
$$

which is well known. For results concerning the skewed Wright-Fisher model we refer the reader to Section 5 .

Example 4.5. (Dirichlet model) Suppose that $\phi(z)=-\log (1-z),|z|<1$, i.e. $\phi_{m}=(m-1) !, m \in \mathbb{N}$. Then, $\sigma_{k}(\theta)=[\theta]_{k}, k \in \mathbb{N}$, and, hence,

$$
\widehat{P}_{i, j}=\frac{(N)_{j}}{[\theta N]_{i}} B_{i, j}([\theta] .)=\frac{i !}{[\theta N]_{i}}\left(\begin{array}{c}
N \\
j
\end{array}\right) \sum_{\substack{l_{1}, \ldots, l_{j} \in \mathbb{N} \\
l_{1}+\cdots+l_{j}=i}} \frac{[\theta]_{l_{1}} \cdots[\theta]_{l_{j}}}{l_{1} ! \cdots l_{j} !} .
$$

Results on the skewed Dirichlet model are presented in Section 6 .

We briefly mention two further examples, showing the wide variety of models we are concerned with.

Example 4.6. Let $\alpha \in(0,1]$ and assume that $\phi(z)=1-(1-z)^{\alpha},|z|<1$. Then, $\phi_{m}=(-1)^{m-1}(\alpha)_{m}=\alpha[1-\alpha]_{m-1} \geq 0, m \in \mathbb{N}$. Note that $\phi(z) \rightarrow 1$ as $z \rightarrow 1$, a smoothness property of $\phi$. For $z \rightarrow 1$ the random variable $\xi_{1}$ has a discrete stable distribution with pgf $\mathbb{E}\left(x^{\xi_{1}}\right)=\exp \left(-\theta(1-x)^{\alpha}\right)$ (see, for example, Steutel and van Harn [33, Eq. (3.7)]) and tail asymptotics $\mathbb{P}\left(\xi_{1}>y\right) \sim \theta c_{\alpha} y^{-\alpha}$ as $y \rightarrow \infty$, where $c_{\alpha}:=\sin (\pi \alpha / 2) \cos (\pi \alpha / 2) \Gamma(\alpha) /(\pi / 2)$ (apply, for example, Pitman [27, Theorem 1]). Note that $\phi_{1}=\alpha$ and that $\phi_{2}=\alpha \beta$ with $\beta:=1-\alpha$. Thus, from (22), it follows that

$$
c_{N}=\widehat{P}_{2,1}=\frac{N\left(\theta \alpha \beta+\theta^{2} \alpha^{2}\right)}{\theta N \alpha \beta+(\theta N)^{2} \alpha^{2}} \sim\left(1+\frac{\beta}{\theta \alpha}\right) \frac{1}{N} .
$$

Thus, $N_{e}=1 / c_{N} \sim \rho N$ with $\rho:=(1+\beta /(\theta \alpha))^{-1}<1$. 
Example 4.7. Let $\alpha \in(0, \infty)$ and assume that $\phi(z)=(1-z)^{-\alpha}-1,|z|<1$. Then, $\phi_{m}=[\alpha]_{m}, m \in \mathbb{N}$. Note that $\phi(z) \rightarrow \infty$ as $z \rightarrow 1$. The random variable $\xi_{1}$ has a compound Poisson distribution with negative binomial jumps and pgf $\mathbb{E}\left(x^{\xi_{1}}\right)=\exp (-\theta \phi(z)(1-\phi(z x) / \phi(z))),|x| \leq 1$. By $(22)$,

$$
c_{N}=\frac{N\left(\theta \alpha(\alpha+1)+\theta^{2} \alpha^{2}\right)}{\theta N \alpha(\alpha+1)+(\theta N)^{2} \alpha^{2}} \sim\left(1+\frac{\alpha+1}{\theta \alpha}\right) \frac{1}{N} .
$$

Thus, $N_{e} \sim \rho N$ with $\rho:=(1+(1+\alpha) /(\theta \alpha))^{-1}<1$.

Theorem 4.3 clarifies that a large class of unbiased conditional branching process models is in the domain of attraction of the Kingman coalescent. For the skewed situation the asymptotical behavior of (17) as $N \rightarrow \infty$ is much more involved. In the following sections we focus on particular skewed population models. It will turn out that these models are not necessarily in the domain of attraction of the Kingman coalescent.

\section{Skewed Wright-Fisher model}

Let $m_{1}, m_{2}, \ldots \in(0, \infty)$ be given parameters and assume that $\xi_{n}$ is Poisson distributed with parameter $m_{n}, n \in \mathbb{N}$. Since $\xi_{n}$ has pgf $f_{n}(x)=\mathbb{E}\left(x^{\xi_{n}}\right)=e^{-m_{n}(1-x)}$, $n \in \mathbb{N}$, it follows that this model belongs to the skewed compound Poisson class (13) with $\phi(z):=z$ and $\theta_{n}:=m_{n} / z$. Moreover, from (9) and (10) it follows that $\mu$ has a multinomial distribution with parameters $N$ and $s_{1, N}, \ldots, s_{N, N}$, where $s_{n, N}:=m_{n} /\left(m_{1}+\cdots+m_{N}\right), n \in\{1, \ldots, N\}$. We shall often drop the index $N$ and use $s_{n}$ instead of $s_{n, N}$ for notational convenience. Note that $\mu$ has pgf $\mathbb{E}\left(z_{1}^{\mu_{1}} \cdots z_{N}^{\mu_{N}}\right)=\left(s_{1} z_{1}+\cdots+s_{N} z_{N}\right)^{N},\left|z_{1}\right|, \ldots,\left|z_{N}\right| \leq 1$, and descending factorial moments $\mathbb{E}\left(\left(\mu_{1}\right)_{l_{1}} \cdots\left(\mu_{N}\right)_{l_{N}}\right)=(N)_{l} s_{1}^{l_{1}} \cdots s_{N}^{l_{N}}, l_{1}, \ldots, l_{N} \in \mathbb{N}_{0}$, where $l:=l_{1}+\cdots+l_{N}$. The pgf of the $n$th marginal $\mu_{n}$ is $\mathbb{E}\left(z_{n}^{\mu_{n}}\right)=\left(1-s_{n}+z_{n} s_{n}\right)^{N}$, $\left|z_{n}\right| \leq 1$, showing that $\mu_{n}$ has a binomial distribution with parameters $N$ and $s_{n}$. In particular, $\mathbb{E}\left(\mu_{n}\right)=N s_{n}$ and $\sigma_{n}^{2}:=\operatorname{Var}\left(\mu_{n}\right)=N s_{n}\left(1-s_{n}\right), n \in\{1, \ldots, N\}$. From the above formula for the descending factorial moments of $\mu$, it follows that $\mathbb{E}\left(\mu_{n_{1}} \mu_{n_{2}}\right)=N(N-1) s_{n_{1}} s_{n_{2}}$ for $n_{1}, n_{2} \in\{1, \ldots, N\}$ with $n_{1} \neq n_{2}$. In particular, $\operatorname{Cov}\left(\mu_{n_{1}}, \mu_{n_{2}}\right)=-N s_{n_{1}} s_{n_{2}}$ for $n_{1} \neq n_{2}$.

The expression (11) for the forward transition probabilities $P_{i, j}$ simplifies to

$$
P_{i, j}=\frac{1}{\left(\begin{array}{c}
N \\
i
\end{array}\right)} \sum_{1 \leq n_{1}<\cdots<n_{i} \leq N}\left(\begin{array}{c}
N \\
j
\end{array}\right)\left(\sum_{k=1}^{i} s_{n_{k}}\right)^{j}\left(1-\sum_{k=1}^{i} s_{n_{k}}\right)^{N-j}, \quad i, j \in S .
$$

Similarly, (12) reduces to

$$
\widehat{P}_{i, j}=i ! \sum_{\substack{l_{1}, \ldots, l_{j} \in \mathbb{N} \\ l_{1}+\cdots+l_{j}=i}} \frac{1}{l_{1} ! \cdots l_{j} !} \sum_{1 \leq n_{1}<\cdots<n_{j} \leq N} s_{n_{1}}^{l_{1}} \cdots s_{n_{j}}^{l_{j}}, \quad i, j \in S,
$$

which, for $j=1$, yields $\widehat{P}_{i, 1}=\sum_{n=1}^{N} s_{n}^{i}, i \in\{1, \ldots, N\}$. For $i=2$ and $i=3$, we obtain $c_{N}=\widehat{P}_{2,1}=\sum_{n=1}^{N} s_{n}^{2}$ and $d_{N}=\widehat{P}_{3,1}=\sum_{n=1}^{N} s_{n}^{3}$. Note that $c_{N}=$ $\sum_{n=1}^{N} s_{n}^{2} \geq 1 / N$. In particular, $N_{e}:=1 / c_{N} \leq N$, so the effective population size is smaller than or equal to the effective population size in the unbiased case. Applying the Hölder inequality $\sum_{n}\left|a_{n} b_{n}\right| \leq\left(\sum_{n} a_{n}^{2}\right)^{1 / 2}\left(\sum_{n} b_{n}^{2}\right)^{1 / 2}$ with $a_{n}:=s_{n}^{1 / 2}$ and 
$b_{n}:=s_{n}^{3 / 2}$ shows that $c_{N} \leq d_{N}^{1 / 2}$, or, equivalently, that $c_{N} \leq d_{N} / c_{N}$. On the other hand, since the Euclidian 2-norm is larger than or equal to the Euclidian 3-norm, we obtain $c_{N}^{1 / 2} \geq d_{N}^{1 / 3}$, or, equivalently, $d_{N} / c_{N} \leq c_{N}^{1 / 2}$. Thus, $c_{N} \leq d_{N} / c_{N} \leq c_{N}^{1 / 2}$ and, consequently, $c_{N} \rightarrow 0$ is equivalent to $d_{N} / c_{N} \rightarrow 0$. The model is hence in the domain of attraction of the Kingman coalescent if and only if $c_{N} \rightarrow 0$.

Choosing $i=j$ in (24) shows that the backward matrix $\widehat{P}$ has eigenvalues $\lambda_{0}=1$ and

$$
\lambda_{i}:=\widehat{P}_{i, i}=i ! \sum_{1 \leq n_{1}<\cdots<n_{i} \leq N} s_{n_{1}} \cdots s_{n_{i}}, \quad i \in\{1, \ldots, N\}
$$

By duality [20], these are also the eigenvalues of the forward transition matrix $P$. Note that $1=\lambda_{0}=\lambda_{1}>\lambda_{2}=1-c_{N}>\lambda_{3}>\cdots>\lambda_{N}$. In particular, the eigenvalues $\lambda_{1}, \ldots, \lambda_{N}$ are pairwise distinct. Coming back to the random variables $\mu_{1}, \ldots, \mu_{N}$ we see that the average covariances of the $\mu_{n}$ 's is

$$
\frac{1}{\left(\begin{array}{c}
N \\
2
\end{array}\right)} \sum_{1 \leq n_{1}<n_{2} \leq N} \operatorname{Cov}\left(\mu_{n_{1}}, \mu_{n_{2}}\right)=-\frac{2}{N-1} \sum_{1 \leq n_{1}<n_{2} \leq N} s_{n_{1}} s_{n_{2}}=-\frac{1-c_{N}}{N-1} .
$$

Let

$$
S_{N}:=\frac{1}{N} \sum_{n=1}^{N} \frac{\mathbb{E}\left(\mu_{n}\right)}{N} \mu_{n}=\frac{1}{N} \sum_{n=1}^{N} s_{n} \mu_{n}
$$

be the size-biased relative mean offspring fertility. Since $\mu$ has a multinomial distribution with parameters $N$ and $s_{1}, \ldots, s_{N}$, it is readily checked that $\mathbb{E}\left(S_{N}\right)=c_{N}$ and that $\operatorname{Var}\left(S_{N}\right)=\left(d_{N}-c_{N}^{2}\right) / N$, which provides an alternative proof of the inequality $d_{N} \geq c_{N}^{2}$.

For the Wright-Fisher model, the moments of $X_{t+1}$, given $X_{t}=i$, can be related to the backward probabilities as follows. For $j \in \mathbb{N}$, we have

$$
\begin{aligned}
\mathbb{E}\left(X_{t+1}^{j} \mid X_{t}=i\right) & =\frac{1}{\left(\begin{array}{c}
N \\
i
\end{array}\right)} \sum_{1 \leq n_{1}<\cdots<n_{i} \leq N} \mathbb{E}\left(\left(\sum_{k=1}^{i} \mu_{n_{k}}\right)^{j}\right) \\
& =\frac{1}{\left(\begin{array}{c}
N \\
i
\end{array}\right)} \sum_{l=1}^{j} S(l, j)(N)_{l} \sum_{1 \leq n_{1}<\cdots<n_{i} \leq N}\left(\sum_{k=1}^{i} s_{n_{k}}\right)^{l} .
\end{aligned}
$$

Using

$$
\sum_{1 \leq n_{1}<\cdots<n_{i} \leq N}\left(\sum_{k=1}^{i} s_{n_{k}}\right)^{l}=\sum_{k=1}^{l}\left(\begin{array}{c}
N-k \\
i-k
\end{array}\right) \widehat{P}_{l, k},
$$

where $\widehat{P}_{l, k}$ is defined in (24), we get

$$
\begin{aligned}
\mathbb{E}\left(X_{t+1}^{j} \mid X_{t}=i\right) & =\frac{1}{\left(\begin{array}{c}
N \\
i
\end{array}\right)} \sum_{l=1}^{j} S(l, j)(N)_{l} \sum_{k=1}^{l}\left(\begin{array}{c}
N-k \\
i-k
\end{array}\right) \widehat{P}_{l, k} \\
& =\sum_{l=1}^{j} S(l, j)(N)_{l} \sum_{k=1}^{l} \frac{(i)_{k}}{(N)_{k}} \widehat{P}_{l, k},
\end{aligned}
$$

which is a polynomial of degree $j$ in $i$.

For the Wright-Fisher model, there are the following two alternative representations of the backward transition probabilities (24). 
(i) It can easily be checked that

$$
\widehat{P}_{i, j}=N^{-i}(N)_{j} S(i, j, N s),
$$

where $s:=\left(s_{1}, \ldots, s_{N}\right)$ and

$$
S(i, j, s):=\frac{i !}{j !}\left[x^{i}\right] \frac{1}{\left(\begin{array}{c}
N \\
j
\end{array}\right)} \sum_{1 \leq n_{1}<\cdots<n_{j} \leq N} \prod_{k=1}^{j}\left(e^{x s_{n_{k}}}-1\right)
$$

are generalized second kind Stirling numbers. Note that, when $\mathbb{E}\left(\mu_{n}\right)=N s_{n}=1$ for all $n \in[N],(26)$ reduces to the usual second kind Stirling numbers $S(i, j)=$ $(i ! / j !)\left[x^{i}\right]\left(e^{x}-1\right)^{j}$ (see $[5$, vol. I, p. 144]). The unbiased version of $(25)$ is thus $\widehat{P}_{i, j}=N^{-i}(N)_{j} S(i, j)$, which is well-known. Note that (25) and (26) also yield

$$
\widehat{P}_{i, j}=i !\left[x^{i} z^{j}\right] \prod_{n=1}^{N}\left(1+z\left(e^{x s_{n}}-1\right)\right)
$$

showing that, as shown in [18],

$$
\mathbb{E}\left(z^{\widehat{X}_{t+1}} \mid \widehat{X}_{t}=i\right)=i !\left[x^{i}\right] \prod_{n=1}^{N}\left(1+z\left(e^{x s_{n}}-1\right)\right),
$$

and for the double pgf

$$
\sum_{i} \frac{x^{i}}{i !} \mathbb{E}\left(z^{\widehat{X}_{t+1}} \mid \widehat{X}_{t}=i\right)=\prod_{n=1}^{N}\left(1+z\left(e^{x s_{n}}-1\right)\right) .
$$

This leads in particular to $\mathbb{E}\left(\widehat{X}_{t+1} \mid \widehat{X}_{t}=i\right)=\sum_{n=1}^{N}\left(1-\left(1-s_{n}\right)^{i}\right)$ and

$$
\begin{aligned}
& \mathbb{E}\left(\left(\begin{array}{c}
\widehat{X}_{t+1} \\
2
\end{array}\right) \mid \widehat{X}_{t}=i\right) \\
& \quad=\sum_{n=1}^{N}(n-1)\left(1-\left(1-s_{n}\right)^{i}\right)-\sum_{1 \leq n_{2}<n_{1} \leq N}\left(\left(1-s_{n_{1}}\right)^{i}-\left(1-\left(s_{n_{1}}+s_{n_{2}}\right)\right)^{i}\right) .
\end{aligned}
$$

(ii) There is a second obvious representation taking into account repetitions, in the spirit of the Ewens sampling formula [8]. Assume there are $a_{l}$ individuals at generation $t$, numbered $1 \leq n_{1, l}<\cdots<n_{a_{l}, l} \leq N$, producing exactly $l$ offspring, $l \in\{0, \ldots, N\}$. Clearly, there is no overlap of the above number of sequences for different values of $l$. Then, using (24)

$$
\widehat{P}_{i, j}=i ! \sum_{\substack{\sum_{l=1}^{i} l a_{l}=i ; \\ \sum_{l=1}^{i} a_{l}=j}} \sum_{l=1}^{i} \sum_{n_{1, l}<\cdots<n_{a_{l}, l}} \prod_{l=1}^{i} \prod_{k=1}^{a_{l}}\left(\frac{s_{n_{k, l}}^{l}}{l !}\right) .
$$

In $(27)$, there are

$$
\prod_{l=1}^{i}\left(\begin{array}{c}
N-\sum_{k=1}^{l-1} a_{k} \\
a_{l}
\end{array}\right)=\frac{(N)_{j}}{\prod_{l=1}^{i} a_{l} !}
$$


sums of the type $\sum_{l=1}^{i} \sum_{n_{1, l}<\cdots<n_{a_{l}, l}} 1$ so that when $s_{n}=1 / N$ for all $n \in[N]$ (the unbiased case),

$$
\widehat{P}_{i, j}=N^{-i}(N)_{j} \sum_{\substack{\sum_{l=1}^{i} l a_{l}=i ; \\ \sum_{l=1}^{i} a_{l}=j}} \frac{i !}{\prod_{l=1}^{i} a_{l} ! l ! a^{l}}
$$

where the last sum is an alternative representation of the second kind Stirling numbers $S(i, j)$ (see [5, vol. I, p. 145]).

Example 5.1. (power law growth) Fix a constant $\alpha \in \mathbb{R}$ and assume that $m_{n}:=$ $\mathbb{E}\left(\xi_{n}\right)=n^{-\alpha}, n \in \mathbb{N}$. The unbiased case $\left(m_{n}=1\right.$ for all $\left.n \in \mathbb{N}\right)$ corresponds to $\alpha=0$. In the following seven ranges for the parameter $\alpha$ are distinguished.

(i) If $\alpha<1 / 3$, then $M_{N}:=\sum_{n=1}^{N} m_{n} \sim N \int_{0}^{1}(N x)^{-\alpha} d x=N^{1-\alpha} /(1-\alpha)$,

$$
c_{N}=\frac{1}{M_{N}^{2}} \sum_{n=1}^{N} n^{-2 \alpha} \sim\left(\frac{1-\alpha}{N^{1-\alpha}}\right)^{2} \frac{N^{1-2 \alpha}}{1-2 \alpha}=\frac{(1-\alpha)^{2}}{(1-2 \alpha) N}
$$

and, similarly,

$$
d_{N}=\frac{1}{M_{N}^{3}} \sum_{n=1}^{N} n^{-3 \alpha} \sim\left(\frac{1-\alpha}{N^{1-\alpha}}\right)^{3} \frac{N^{1-3 \alpha}}{1-3 \alpha}=\frac{(1-\alpha)^{3}}{1-3 \alpha} \frac{1}{N^{2}} .
$$

(ii) If $\alpha=1 / 3$, then (28) still holds, i.e. $c_{N} \sim 4 /(3 N)$, but

$$
d_{N}=\frac{1}{M_{N}^{3}} \sum_{n=1}^{N} \frac{1}{n} \sim\left(\frac{1-\alpha}{N^{1-\alpha}}\right)^{3} \log N=\frac{8}{27} \frac{\log N}{N^{2}} .
$$

(iii) If $1 / 3<\alpha<1 / 2$, then (28) still holds, but

$$
d_{N}=\frac{1}{M_{N}^{3}} \sum_{n=1}^{N} n^{-3 \alpha} \sim\left(\frac{1-\alpha}{N^{1-\alpha}}\right)^{3} \zeta(3 \alpha)=(1-\alpha)^{3} \zeta(3 \alpha) \frac{1}{N^{3(1-\alpha)}},
$$

where $\zeta$ denotes the Riemann zeta function.

In all three cases, i.e. for $\alpha<1 / 2$, we have $N_{e}=1 / c_{N} \sim \rho N$ with $0<\rho:=$ $(1-2 \alpha) /(1-\alpha)^{2} \leq 1$. The effective population size is hence asymptotically of a factor $\rho$ smaller than the effective population size $N$ in the unbiased case $(\alpha=0)$.

Moreover, from (24), it follows that the eigenvalues satisfy

$$
\lambda_{i}=\widehat{P}_{i, i}=\frac{i !}{M_{N}^{i}} \sum_{1 \leq n_{1}<\cdots<n_{i} \leq N}\left(n_{1} \cdots n_{i}\right)^{-\alpha} \sim\left(\frac{1-\alpha}{N^{1-\alpha}}\right)^{i} i ! \sum_{n=i !}^{(N)_{i}} n^{-\alpha} M_{N}(n, i),
$$

where $M_{N}(n, i)$ is the number of multiplicative partitions of $n$ into $i$ ordered distinct factors each belonging to $\{1, \ldots, N\}$.

We will now see that $N_{e}$ can increase of order slower than $N$.

(iv) When $\alpha=1 / 2$, it is readily checked that $M_{N} \sim 2 N^{1 / 2}, c_{N} \sim(\log N) /(4 N)$, and $d_{N} \sim \zeta(3 / 2) /\left(8 N^{3 / 2}\right)$. Thus, $N_{e}=1 / c_{N} \sim(4 N) / \log N$ is asymptotically of a factor $4 / \log N$ smaller than the standard effective population size $N$ in the unbiased case. 
(v) Assume now that $1 / 2<\alpha<1$. Then, still $M_{N}=\sum_{n=1}^{N} n^{-\alpha} \sim N^{1-\alpha} /(1-\alpha)$,

$$
c_{N}=\frac{1}{M_{N}^{2}} \sum_{n=1}^{N} n^{-2 \alpha} \sim \frac{(1-\alpha)^{2}}{N^{2(1-\alpha)}} \zeta(2 \alpha)
$$

and, similarly,

$$
d_{N}=\frac{1}{M_{N}^{3}} \sum_{n=1}^{N} n^{-3 \alpha} \sim \frac{(1-\alpha)^{3}}{N^{3(1-\alpha)}} \zeta(3 \alpha)
$$

Note that, with $\rho:=1 /\left((1-\alpha)^{2} \zeta(2 \alpha)\right)<1, N_{e} \sim \rho N^{2(1-\alpha)}$ grows algebraically and the order is slower than $N$.

(vi) For $\alpha=1$ it is straightforward to check that $M_{N} \sim \log N, c_{N} \sim \zeta(2) /(\log N)^{2}$, and $d_{N} \sim \zeta(3) /(\log N)^{3}$. Note that $N_{e} \sim(\log N)^{2} / \zeta(2)$ grows quite slow (logarithmically).

For all six cases (i) - (vi) considered so far, i.e. for $\alpha \leq 1$, we have $c_{N} \rightarrow 0$ and $d_{N} / c_{N} \rightarrow 0$. Thus, in the sense of Definition $2.1 \mathrm{a}$ ), the model is in the domain of attraction of the Kingman coalescent. Thus, for each sample size $n \in \mathbb{N}$, the time-scaled backward process $\left(\mathcal{R}_{\left[t / c_{N}\right]}^{(n)}\right)_{t \in[0, \infty)}$ converges weakly to the Kingman $n$ coalescent as $N \rightarrow \infty$. All that is left about the details of the original discrete fertility model $\left(m_{n}=n^{-\alpha}\right)$ is enclosed in the parameter $N_{e}=1 / c_{N} \leq N$, where $N$ is the time-scale of the unbiased Wright-Fisher model $\left(m_{n}=1\right)$.

(vii) Assume now that $\alpha>1$. Then, $M_{N}=\sum_{n=1}^{N} n^{-\alpha} \rightarrow \zeta(\alpha)=: M$, and,

$$
\widehat{P}_{i, 1}=\Phi_{1}(i)=\sum_{n=1}^{N} s_{n}^{i}=\frac{1}{M_{N}^{i}} \sum_{n=1}^{N} n^{-i \alpha} \rightarrow \frac{\zeta(i \alpha)}{(\zeta(\alpha))^{i}}, \quad i \in \mathbb{N} .
$$

In particular $c_{N} \rightarrow \zeta(2 \alpha) /(\zeta(\alpha))^{2}>0$ and $d_{N} \rightarrow \zeta(3 \alpha) /(\zeta(\alpha))^{3}>0$. The regime $\alpha>1$ thus differs significantly from the previously studied cases, since $c_{N}$ converges to a positive constant as $N \rightarrow \infty$. More generally, for $l_{1}, \ldots, l_{j} \in \mathbb{N}$, we have to analyze the behavior of

$$
\Phi_{j}^{(N)}\left(l_{1}, \ldots, l_{j}\right)=\sum_{\substack{n_{1}, \ldots, n_{j}=1 \\ \text { all distinct }}}^{N} s_{n_{1}, N}^{l_{1}} \cdots s_{n_{j}, N}^{l_{j}}
$$

as $N \rightarrow \infty$. For each fixed $n \in \mathbb{N}$ we have $s_{n, N}=n^{-\alpha} / M_{N} \rightarrow n^{-\alpha} / M=$ $n^{-\alpha} / \zeta(\alpha)=: p_{n}$ as $N \rightarrow \infty$. Note that $\sum_{n=1}^{\infty} p_{n}=1$. Moreover, $s_{n, N} \leq n^{-\alpha}$ uniformly for all $N$, since $M_{N} \geq 1$. Thus, by dominated convergence, for each $l_{1}, \ldots, l_{j} \in \mathbb{N}$, the limit $\phi_{j}\left(l_{1}, \ldots, l_{j}\right):=\lim _{N \rightarrow \infty} \Phi_{j}^{(N)}\left(l_{1}, \ldots, l_{j}\right)$ exists and is of the form

$$
\phi_{j}\left(l_{1}, \ldots, l_{j}\right)=\sum_{\substack{n_{1}, \ldots, n_{j} \in \mathbb{N} \\ \text { all distinct }}} p_{n_{1}}^{l_{1}} \cdots p_{n_{j}}^{l_{j}} .
$$

For arbitrary $i, j \in \mathbb{N}$ it therefore follows from (24) that

$$
\lim _{N \rightarrow \infty} \widehat{P}_{i, j}=\frac{i !}{j !} \sum_{\substack{l_{1}, \ldots, l_{j} \in \mathbb{N} \\ l_{1}+\cdots+l_{j}=i}} \frac{\phi_{j}\left(l_{1}, \ldots, l_{j}\right)}{l_{1} ! \cdots l_{j} !} .
$$


It is convenient (see, Schweinsberg [31]) to rewrite (29) in integral form as

$$
\phi_{j}\left(l_{1}, \ldots, l_{j}\right)=\int_{\Delta} \sum_{\substack{n_{1}, \ldots, n_{j} \in \mathbb{N} \\ \text { all distinct }}} x_{n_{1}}^{l_{1}} \cdots x_{n_{j}}^{l_{j}} \frac{\Xi(d x)}{(x, x)},
$$

where $\Delta:=\left\{\left(x_{1}, x_{2}, \ldots\right): x_{1} \geq x_{2} \geq \cdots \geq 0, \sum_{n=1}^{\infty} x_{n} \leq 1\right\},(x, x):=\sum_{n=1}^{\infty} x_{n}^{2}$ for $x \in \Delta$ and the measure $\Xi$ on $\Delta$ assigns its total mass $\Xi(\Delta):=(p, p)=\sum_{n=1}^{\infty} p_{n}^{2}=$ $\zeta(2 \alpha) /(\zeta(\alpha))^{2}$ to the single point $p=\left(p_{1}, p_{2}, \ldots\right) \in \Delta$. Theorem 2.1 of [24] ensures that the model, without any time-scaling involved, is in the domain of attraction (in the sense of Definition $2.1 \mathrm{~b}$ )) of the discrete-time $\Xi$-coalescent with the measure $\Xi$ as just defined.

Example 5.2. (geometric growth) Fix a constant $\lambda \in(0, \infty)$ and assume that $m_{n}=\lambda^{n}, n \in \mathbb{N}$. The unbiased case corresponds to $\lambda=1$, so without loss of generality we assume that $\lambda \neq 1$. The distribution of $\mu$ when $\lambda>1$ is exactly the same as the distribution of $\mu$ when the parameter is $1 / \lambda$, just with the order of the $N$ associated random variables $\mu_{1}, \ldots, \mu_{N}$ reversed. Both distributions are multinomial with the same parameters. Therefore, without loss of generality, we do the calculations only for $\lambda \in(0,1)$. Then, $M_{N}=\sum_{n=1}^{N} \lambda^{n}=\lambda\left(1-\lambda^{N}\right) /(1-\lambda) \rightarrow$ $\lambda /(1-\lambda)=: M$ as $N \rightarrow \infty$ and

$$
\begin{aligned}
\widehat{P}_{i, 1} & =\frac{1}{M_{N}^{i}} \sum_{n=1}^{N} \lambda^{i n}=\left(\frac{1-\lambda}{\lambda\left(1-\lambda^{N}\right)}\right)^{i} \frac{\lambda^{i}\left(1-\lambda^{i N}\right)}{1-\lambda^{i}} \\
& =\frac{(1-\lambda)^{i}}{1-\lambda^{i}} \frac{1-\lambda^{i N}}{\left(1-\lambda^{N}\right)^{i}} \sim \frac{(1-\lambda)^{i}}{1-\lambda^{i}} .
\end{aligned}
$$

In particular,

$$
c_{N}=\widehat{P}_{2,1}=\frac{(1-\lambda)^{2}}{1-\lambda^{2}} \frac{1-\lambda^{2 N}}{\left(1-\lambda^{N}\right)^{2}} \sim \frac{1-\lambda}{1+\lambda} .
$$

For arbitrary $i, j \in \mathbb{N}$ it follows similarly as in the previous Example 5.1 (vii) that the limiting formula (30) for $\widehat{P}_{i, j}$ holds, but in the formula $(29)$ for $\phi_{j}\left(l_{1}, \ldots, l_{j}\right)$, the parameter $p_{n}$ has to be replaced by $p_{n}:=\lambda^{n} / M=(1-\lambda) \lambda^{n-1}, n \in \mathbb{N}$. For instance, the diagonal entries $\widehat{P}_{i, i}$ of the matrix $\widehat{P}$ satisfy

$$
\begin{aligned}
\lim _{N \rightarrow \infty} \widehat{P}_{i, i} & =\phi_{i}(1, \ldots, 1)=\sum_{\substack{n_{1}, \ldots, n_{i} \in \mathbb{N} \\
\text { all distinct }}} p_{n_{1}} \cdots p_{n_{i}} \\
& =\left(\frac{1-\lambda}{\lambda}\right)^{i} \sum_{\substack{n_{1}, \ldots, n_{i} \in \mathbb{N} \\
\text { all distinct }}} \lambda^{n_{1}+\cdots+n_{i}}=\left(\frac{1-\lambda}{\lambda}\right)^{i} \sum_{n \geq i(i+1) / 2} \lambda^{n} A(n, i),
\end{aligned}
$$

where $A(n, i)$ is the number of vectors $\left(n_{1}, \ldots, n_{i}\right) \in \mathbb{N}^{i}$ with pairwise distinct components satisfying $n_{1}+\cdots+n_{i}=n$. This example essentially coincides with Example 5.1 (vii). Again (see [24, Theorem 2.1]) the model is the domain of attraction (in the sense of Definition $2.1 \mathrm{~b}$ )) of a discrete-time $\Xi$-coalescent, where the measure $\Xi$ assigns its total mass $\Xi(\Delta)=(p, p)=(1-\lambda) /(1+\lambda)$ to the single point $p:=\left(p_{1}, p_{2}, \ldots\right) \in \Delta$. The same result holds for $\lambda>1$, but (see the comment at the beginning of this example) in the definition of $p_{n}$ above, the parameter $\lambda$ has to be replaced by $1 / \lambda$. 


\section{Skewed Dirichlet MOdel}

Consider the model where $\xi_{n}$ has a negative binomial distribution with pgf $f_{n}(x)=$ $(p /(1-q x))^{a_{n}}$, where $a_{n}>0, p \in(0,1), q:=1-p$. Note that $m_{n}:=\mathbb{E}\left(\xi_{n}\right)=$ $(q / p) a_{n}, n \in \mathbb{N}$, and that this model belongs to the skewed compound Poisson class (13) with $\theta_{n}:=a_{n}, \phi(z):=-\log (1-z)=\sum_{m=1}^{\infty} z^{m} / m$, and $z:=q$. In this case $\mu$ has the Dirichlet multinomial distribution

$$
\mathbb{P}(\mu=k)=\frac{\left(\begin{array}{c}
a_{1}+k_{1}-1 \\
k_{1}
\end{array}\right) \cdots\left(\begin{array}{c}
a_{N}+k_{N}-1 \\
k_{N}
\end{array}\right)}{\left(\begin{array}{c}
A_{N}+N-1 \\
N
\end{array}\right)}=\frac{N !}{k_{1} ! \cdots k_{N} !} \frac{\left[a_{1}\right]_{k_{1}} \cdots\left[a_{N}\right]_{k_{N}}}{\left[A_{N}\right]_{N}},
$$

$k=\left(k_{1}, \ldots, k_{N}\right) \in \Delta(N)$, where $A_{N}:=a_{1}+\cdots+a_{N}$. In other words, the distribution of $\mu$ is multinomial with parameters $N$ and $p_{1}, \ldots, p_{N}$, where the parameters $p_{1}, \ldots, p_{N}$ are random and Dirichlet distributed with parameters $a_{1}, \ldots, a_{N}$. The symmetric Dirichlet model is for example studied in [29, p. 847]. From (11), it follows that the forward process $X$ has transition probabilities

$$
P_{i, j}=\frac{1}{\left(\begin{array}{c}
N \\
i
\end{array}\right)} \sum_{1 \leq n_{1}<\cdots<n_{j} \leq N} \frac{\left(\begin{array}{c}
a_{n_{1}}+\cdots+a_{n_{j}} \\
j
\end{array}\right)\left(\begin{array}{c}
A_{N}-\left(a_{n_{1}}+\cdots+a_{n_{j}}\right) \\
N-j
\end{array}\right)}{\left(\begin{array}{c}
A_{N}+N-1 \\
N
\end{array}\right)}, \quad i, j \in S .
$$

Note that $\mu$ has joint descending factorial moments

$$
\mathbb{E}\left(\left(\mu_{1}\right)_{l_{1}} \cdots\left(\mu_{N}\right)_{l_{N}}\right)=\frac{(N)_{l}}{\left[A_{N}\right]_{l}} \prod_{n=1}^{N}\left[a_{n}\right]_{l_{n}}, \quad l_{1}, \ldots, l_{N} \in \mathbb{N}_{0},
$$

where $l:=l_{1}+\cdots+l_{N}$. In particular, $\mathbb{E}\left(\mu_{n}\right)=N a_{n} / A_{N}, n \in\{1, \ldots, N\}$. We conclude from (12) that the backward process $\widehat{X}$ has transition probabilities

$$
\widehat{P}_{i, j}=\frac{i !}{\left[A_{N}\right]_{i}} \sum_{1 \leq n_{1}<\cdots<n_{j} \leq N} \sum_{\substack{l_{1}, \ldots, l_{j} \in \mathbb{N} \\ l_{1}+\cdots+l_{j}=i}} \prod_{k=1}^{j} \frac{\left[a_{n_{k}}\right]_{l_{k}}}{l_{k} !}, \quad i, j \in S,
$$

with the convention that $\widehat{P}_{i, 0}=\delta_{i 0}$. In particular, $\widehat{P}_{i, 1}=\left(\left[A_{N}\right]_{i}\right)^{-1} \sum_{n=1}^{N}\left[a_{n}\right]_{i}$, $i \in\{1, \ldots, N\}$, and hence, $c_{N}=\widehat{P}_{2,1}=\left(\left[A_{N}\right]_{2}\right)^{-1} \sum_{n=1}^{N}\left[a_{n}\right]_{2}>0$. From $a_{n} / A_{N} \leq$ $\left(a_{n}+1\right) /\left(A_{N}+1\right)$ it follows that $c_{N} \geq \sum_{n=1}^{N}\left(a_{n} / A_{n}\right)^{2} \geq 1 / N$, or, equivalently, $N_{e}=1 / c_{N} \leq N$. Moreover, $d_{N}=\widehat{P}_{3,1}=\left(\left[A_{N}\right]_{3}\right)^{-1} \sum_{n=1}^{N}\left[a_{n}\right]_{3}>0$.

It is now verified that $c_{N} \rightarrow 0$ if and only if $d_{N} / c_{N} \rightarrow 0$. The basic idea of the proof is the same as for the skewed Kimura model, however, the technical details are a bit more involved. We have

$$
\max _{1 \leq n \leq N}\left(\frac{a_{n}}{A_{N}}\right)^{2} \leq \sum_{n=1}^{N}\left(\frac{a_{n}}{A_{N}}\right)^{2} \leq c_{N}
$$


Thus, $\max _{1 \leq n \leq N}\left(a_{n} / A_{N}\right) \leq c_{N}^{1 / 2}$. Moreover,

$$
\begin{aligned}
d_{N} & =\sum_{n=1}^{N} \frac{a_{n}\left(a_{n}+1\right)\left(a_{n}+2\right)}{A_{N}\left(A_{N}+1\right)\left(A_{N}+2\right)} \leq \sum_{n=1}^{N} \frac{a_{n}\left(a_{n}+1\right)}{A_{N}\left(A_{N}+1\right)}\left(\frac{a_{n}}{A_{N}}+\frac{2}{A_{N}}\right) \\
& \leq\left(\max _{1 \leq n \leq N} \frac{a_{n}}{A_{N}}\right) \sum_{n=1}^{N} \frac{a_{n}\left(a_{n}+1\right)}{A_{N}\left(A_{N}+1\right)}+\frac{2}{A_{N}} \sum_{n=1}^{N} \frac{a_{n}\left(a_{n}+1\right)}{A_{N}\left(A_{N}+1\right)} \\
& =\left(\max _{1 \leq n \leq N} \frac{a_{n}}{A_{N}}\right) c_{N}+\frac{2}{A_{N}} c_{N} \leq c_{N}^{3 / 2}+\frac{2}{A_{N}} c_{N} .
\end{aligned}
$$

Thus, $d_{N} / c_{N} \leq c_{N}^{1 / 2}+2 / A_{N}$. Suppose now that $c_{N} \rightarrow 0$. From the formula for $c_{N}$ it is readily seen that $c_{N} \geq a_{1}^{2} /\left[A_{N}\right]_{2}$. Thus, $\left[A_{N}\right]_{2} \geq a_{1}^{2} / c_{N} \rightarrow \infty$. It follows that $A_{N} \rightarrow \infty$ and, hence, $d_{N} / c_{N} \leq c_{N}^{1 / 2}+2 / A_{N} \rightarrow 0$. Conversely, if $d_{N} / c_{N} \rightarrow 0$, then $c_{N} \rightarrow 0$ (see Section 2). Thus, $c_{N} \rightarrow 0$ if and only if $d_{N} / c_{N} \rightarrow 0$.

Example 6.1. (power law growth) Suppose that $a_{n}:=n^{-\alpha}$ for some constant $\alpha \in \mathbb{R}$. Five ranges for the parameter $\alpha$ are distinguished. For $\alpha \in(-\infty, 0)$, $A_{N} \sim N^{1-\alpha} /(1-\alpha) \rightarrow \infty$,

$$
c_{N} \sim \frac{1}{A_{N}^{2}} \sum_{n=1}^{N} a_{n}^{2} \sim \frac{(1-\alpha)^{2}}{1-2 \alpha} \frac{1}{N} \quad \text { and } \quad d_{N} \sim \frac{1}{A_{N}^{3}} \sum_{n=1}^{N} a_{n}^{3} \sim \frac{(1-\alpha)^{3}}{1-3 \alpha} \frac{1}{N^{2}} .
$$

For $\alpha=0$ we have $c_{N}=2 /(N+1) \sim 2 / N$ and $d_{N}=6 /((N+1)(N+2)) \sim 6 / N^{2}$. If $\alpha \in(0,1)$, then $c_{N} \sim 1 / A_{N} \sim(1-\alpha) / N^{1-\alpha}$ and $d_{N} \sim 2 / A_{N}^{2} \sim 2(1-\alpha)^{2} / N^{2-2 \alpha}$. If $\alpha=1$, then $c_{N} \sim 1 / \log N \rightarrow 0$ and $d_{N} \sim 2 / \log ^{2} N$. In all these four cases considered so far we have $c_{N} \rightarrow 0$ and $d_{N} / c_{N} \rightarrow 0$, so the model is in the domain of attraction of the Kingman coalescent. Suppose now that $\alpha \in(1, \infty)$. Then, $A_{N}=\sum_{n=1}^{N} n^{-\alpha} \rightarrow A:=\zeta(\alpha)>1, \sum_{n=1}^{N} a_{n}\left(a_{n}+1\right)=\sum_{n=1}^{N}\left(n^{-2 \alpha}+n^{-\alpha}\right) \rightarrow$ $\zeta(2 \alpha)+\zeta(\alpha)$, and, hence, $c_{N} \rightarrow(\zeta(2 \alpha)+\zeta(\alpha)) /[\zeta(\alpha)]_{2}>0$. Similarly, it follows that $d_{N} \rightarrow(\zeta(3 \alpha)+3 \zeta(2 \alpha)+2 \zeta(\alpha)) /[\zeta(\alpha)]_{3}>0$.

For $l_{1}, \ldots, l_{j} \in \mathbb{N} \backslash\{1\}$ and $N \geq i:=l_{1}+\cdots+l_{j}$,

$$
\begin{aligned}
\Phi_{j}\left(l_{1}, \ldots, l_{j}\right) & =\frac{1}{\left[A_{N}\right]_{i}} \sum_{\substack{n_{1}, \ldots, n_{j}=1 \\
\text { all distinct }}}^{N}\left[a_{n_{1}}\right]_{l_{1}} \cdots\left[a_{n_{j}}\right]_{l_{j}} \\
& \rightarrow \frac{1}{[A]_{i}} \sum_{\substack{n_{1}, \ldots, n_{j} \in \mathbb{N} \\
\text { all distinct }}}\left[a_{n_{1}}\right]_{l_{1}} \cdots\left[a_{n_{j}}\right]_{l_{j}}=\sum_{\substack{n_{1}, \ldots, n_{j} \in \mathbb{N} \\
\text { all distinct }}} \mathbb{E}\left(D_{n_{1}}^{l_{1}} \cdots D_{n_{j}}^{l_{j}}\right),
\end{aligned}
$$

where $\left(D_{n_{1}}, \ldots, D_{n_{j}}, 1-\sum_{k=1}^{j} D_{n_{k}}\right)$ has a Dirichlet distribution with parameters $a_{n_{1}}, \ldots, a_{n_{j}}, A-\sum_{k=1}^{j} a_{n_{k}}$. Thus, the limit $\phi_{j}\left(l_{1}, \ldots, l_{j}\right):=\lim _{N \rightarrow \infty} \Phi_{j}\left(l_{1}, \ldots, l_{j}\right)$ exists and is of the form $\phi_{j}\left(l_{1}, \ldots, l_{j}\right)=\int_{\Delta_{j}} x_{1}^{l_{1}} \cdots x_{j}^{l_{j}} M_{j}\left(d x_{1}, \ldots, d x_{j}\right)$, where $\Delta_{j}:=\left\{\left(x_{1}, \ldots, x_{j}\right) \in[0,1]^{j}: x_{1}+\cdots+x_{j} \leq 1\right\}$, and the symmetric measure

$$
M_{j}:=\sum_{\substack{n_{1}, \ldots, n_{j} \in \mathbb{N} \\ \text { all distinct }}} P_{n_{1}, \ldots, n_{j}}
$$

on $\Delta_{j}$ is an infinite mixture of the distributions $P_{n_{1}, \ldots, n_{j}}$ of $\left(D_{n_{1}}, \ldots, D_{n_{j}}\right)$. The measures $M_{1}, M_{2}, \ldots$ completely characterize the limiting discrete-time coalescent process and the model is in the domain of attraction of this coalescent. We now 
determine the characterizing measure $\Xi$ of this coalescent. For $j \in \mathbb{N}$ let $Q_{j}$ denote the probability measure on $\Delta_{j}$ with density

$$
f_{j}(x):=\frac{\Gamma(A)}{\Gamma\left(a_{1}\right) \cdots \Gamma\left(a_{j}\right) \Gamma\left(A-A_{j}\right)} x_{1}^{a_{1}-1} \cdots x_{j}^{a_{j}-1}\left(1-\sum_{k=1}^{j} x_{k}\right)^{A-A_{j}-1},
$$

$x=\left(x_{1}, \ldots, x_{j}\right) \in \Delta_{j}$, with respect to the Lebesgue measure on $\Delta_{j}$. Liouville's integration formula shows that $\int_{0}^{1-\left(x_{1}+\cdots+x_{j}\right)} f_{j+1}\left(x_{1}, \ldots, x_{j+1}\right) d x_{j+1}=f_{j}\left(x_{1}, \ldots, x_{j}\right)$ for $j \in \mathbb{N}$ and $\left(x_{1}, \ldots, x_{j}\right) \in \Delta_{j}$, so the sequence $\left(Q_{j}\right)_{j \in \mathbb{N}}$ is consistent. Kolmogoroff's extension theorem ensures that there exists a probability measure $Q$ on $\mathbb{R}^{\mathbb{N}}$ such that, for each $j \in \mathbb{N}$, the projection of $Q$ on $\mathbb{R}^{j}$ coincides with $Q_{j}$. The probability measure $Q$ is called (see, for example, [11, Section 4]) the Dirichlet-Kingman distribution with parameter $\left(a_{n}\right)_{n \in \mathbb{N}}$. Let $D_{1}, D_{2}, \ldots$ be random variables with joint distribution $Q$ and let $D_{(1)} \geq D_{(2)} \geq \cdots$ denote the $D_{1}, D_{2}, \ldots$ in decreasing order. Note that $D_{n}$ has a beta distribution with parameters $a_{n}$ and $A-a_{n}, n \in \mathbb{N}$. In particular, $\mathbb{E}\left(D_{n}\right)=a_{n} / A$ for $n \in \mathbb{N}$. Now let $\nu$ denote the joint distribution of $\left(D_{(1)}, D_{(2)}, \ldots\right)$ and define $\Xi(d x):=(x, x) \nu(d x)$. Then, for $j, l_{1}, \ldots, l_{j} \in \mathbb{N}$,

$$
\begin{aligned}
\int_{\Delta} \sum_{\substack{n_{1}, \ldots, n_{j} \in \mathbb{N} \\
\text { all distinct }}} x_{n_{1}}^{l_{1}} \cdots x_{n_{j}}^{l_{j}} \frac{\Xi(d x)}{(x, x)}=\sum_{\substack{n_{1}, \ldots, n_{j} \in \mathbb{N} \\
\text { all distinct }}} \mathbb{E}\left(D_{\left(n_{1}\right)}^{l_{1}} \cdots D_{\left(n_{j}\right)}^{l_{j}}\right) \\
=\sum_{\substack{n_{1}, \ldots, n_{j} \in \mathbb{N} \\
\text { all distinct }}} \mathbb{E}\left(D_{n_{1}}^{l_{1}} \cdots D_{n_{j}}^{l_{j}}\right)=\sum_{\substack{n_{1}, \ldots, n_{j} \in \mathbb{N} \\
\text { all distinct }}} \frac{\left[a_{n_{1}}\right]_{l_{1}} \cdots\left[a_{n_{j}}\right]_{l_{j}}}{[A]_{l_{1}+\cdots+l_{j}}}=\phi_{j}\left(l_{1}, \ldots, l_{j}\right),
\end{aligned}
$$

which shows that $\Xi$ is the characterizing measure of this coalescent. Note that for all $M_{j}$-integrable functions $g$ it follows that $\mathbb{E}\left(\sum_{n_{1}, \ldots, n_{j}} g\left(D_{n_{1}}, \ldots, D_{n_{j}}\right)\right)=\int_{\Delta_{j}} g d M_{j}$, where the $\operatorname{sum} \sum_{n_{1}, \ldots, n_{j}}$ extends over all pairwise distinct $n_{1}, \ldots, n_{j} \in \mathbb{N}$. Hence, the measure $M_{j}$, defined in (32), is the $j$ th correlation measure (see, for example, Handa [10, Eq. (2.1)]) of the point process $\sum_{n=1}^{\infty} \delta_{D_{n}}$.

Example 6.2. (geometric growth) Suppose that $a_{n}=\lambda^{n}$ for some $\lambda \in(0, \infty)$. The unbiased case corresponds to $\lambda=1$.

(i) If $\lambda<1$, then $A_{N} \rightarrow A:=\lambda /(1-\lambda)$, so $A_{N}$ converges. We are hence essentially in the situation of Example 6.1 with $\alpha>1$. All results there are valid, but now with $A=\lambda /(1-\lambda)$ (instead of $A=\zeta(\alpha))$ and with $a_{n}=\lambda^{n}$ (instead of $\left.a_{n}=n^{\alpha}\right)$. In particular, the correlation measures $M_{1}, M_{2}, \ldots$ of the limiting coalescent process with simultaneous multiple collisions are again infinite mixtures of the form (32).

(ii) If $\lambda>1$, then $A_{N}=\sum_{n=1}^{N} \lambda^{n} \sim \lambda^{N+1} /(\lambda-1)$, so $A_{N}$ does not converge. We are hence essentially in the situation of Example 5.2 with $\lambda>1$, and it follows that the measure $\Xi$ of the limiting $\Xi$-coalescent assigns its total mass $\Xi(\Delta)=(\lambda-1) /(\lambda+1)$ to the single point $p=\left(p_{1}, p_{2}, \ldots\right) \in \Delta$ defined via $p_{n}:=(\lambda-1) / \lambda^{n}, n \in \mathbb{N}$.

Remark. (Dirichlet-Kingman coalescent) Examples 6.1 and 6.2 (i) lead to the following more general example of a $\Xi$-coalescent. Let $a=\left(a_{n}\right)_{n \in \mathbb{N}}$ be a convergent sequence of positive real numbers. Define $A_{j}:=\sum_{n=1}^{j} a_{n}, j \in \mathbb{N}, A:=$ $\lim _{j \rightarrow \infty} A_{j}=\sum_{n=1}^{\infty} a_{n}$, and, for $j \in \mathbb{N}$, define the function $\phi_{j}: \mathbb{N}^{j} \rightarrow \mathbb{R}$ via

$$
\phi_{j}\left(l_{1}, \ldots, l_{j}\right):=\frac{1}{[A]_{l_{1}+\cdots+l_{j}}} \sum_{\begin{array}{l}
n_{1}, \ldots, n_{j} \in \mathbb{N} \\
\text { all distinct }
\end{array}}\left[a_{n_{1}}\right]_{l_{1}} \cdots\left[a_{n_{j}}\right]_{l_{j}} .
$$


Note that $\phi_{1}(1)=1$ and that the functions $\phi_{j}, j \in \mathbb{N}$, are consistent in the sense that $\phi_{j}\left(l_{1}, \ldots, l_{j}\right)=\phi_{j+1}\left(l_{1}, \ldots, l_{j}, 1\right)+\sum_{i=1}^{j} \phi_{j}\left(l_{1}, \ldots, l_{i-1}, l_{i}+1, l_{i+1}, \ldots, l_{j}\right)$ for all $j, l_{1}, \ldots, l_{j} \in \mathbb{N}$. Thus, there exists a discrete-time $\Xi$-coalescent $R=\left(R_{k}\right)_{k \in \mathbb{N}_{0}}$ such that, if $R$ is in a state with $l \in \mathbb{N}$ blocks, any $\left(l_{1}, \ldots, l_{j}\right)$-collision $\left(j, l_{1}, \ldots, l_{j} \in \mathbb{N}\right.$ with $l_{1}+\cdots+l_{j}=l$ ) occurs with probability (34). The measure $\Xi$ is obtained as described in Example 6.1. More precisely, $\nu(d x):=\Xi(d x) /(x, x)$ is the joint distribution of the ordered random variables $D_{(1)} \geq D_{(2)} \geq \cdots$, where the random variables $D_{1}, D_{2}, \ldots$ are such that, for each $j \in \mathbb{N},\left(D_{1}, \ldots, D_{j}\right)$ has density (33). The random partition $\left(D_{1}, D_{2}, \ldots\right)$ of the unit interval is (see, for example, [11, Section 4]) the Dirichlet-Kingman partition with parameter $a$. One may therefore call $R$ the discrete-time Dirichlet-Kingman coalescent with parameter $a$. A standard method for example well described in [7, p. 163, Eq. (2.3)]) allows to transform the discrete-time coalescent $R$ into a continuous-time coalescent $\Pi=\left(\Pi_{t}\right)_{t \geq 0}$ such that, if this coalescent is in a state with $l \in \mathbb{N}$ blocks, any $\left(l_{1}, \ldots, l_{j}\right)$-collision $\left(j, l_{1}, \ldots, l_{j} \in \mathbb{N}\right.$ with $\left.l_{1}+\cdots+l_{j}=l>j\right)$ occurs at rate (34). Note however that $\Pi$ has total rates $1-\phi_{j}(1, \ldots, 1), j \in \mathbb{N}$. One may call $\Pi$ the continuous-time Dirichlet-Kingman coalescent with parameter $a$. This coalescent has many properties in common with the Poisson-Dirichlet coalescent [22]. For example, both measures $\nu$ and $\Xi$ are concentrated on the subset $\Delta^{*}$ of points $x \in \Delta$ satisfying $|x|:=\sum_{n=1}^{\infty} x_{n}=1$. In particular, $\int|x| \nu(d x)=\nu(\Delta)=1<\infty$, showing that these coalescents, if started in the partition of $\mathbb{N}$ into singletons, do not have proper frequencies and, hence, cannot come down from infinity.

\section{Skewed Kimura model}

In this section an example is presented which (in contrast to the models in Sections 5 and 6) does not belong to the compound Poisson class. Suppose that for each $n \in \mathbb{N}$ the random variable $\xi_{n}$ has a binomial distribution with pgf $f_{n}(x)=(p x+q)^{a_{n}}$, where $a_{n} \in \mathbb{N}, p \in(0,1)$ and $q:=1-p$. Note that $m_{n}:=\mathbb{E}\left(\xi_{n}\right)=p a_{n}, n \in \mathbb{N}$. Then, $\mu=\left(\mu_{1}, \ldots, \mu_{N}\right)$ has a multi-hypergeometric distribution of the form $\mathbb{P}(\mu=$ $k)=\left(\begin{array}{c}a_{1} \\ k_{1}\end{array}\right) \cdots\left(\begin{array}{c}a_{N} \\ k_{N}\end{array}\right) /\left(\begin{array}{c}A_{N} \\ N\end{array}\right), k=\left(k_{1}, \ldots, k_{N}\right) \in \Delta(N)$, where $A_{N}:=a_{1}+\cdots+a_{N}$.

From (11), it follows that the forward process $X$ has transition probabilities

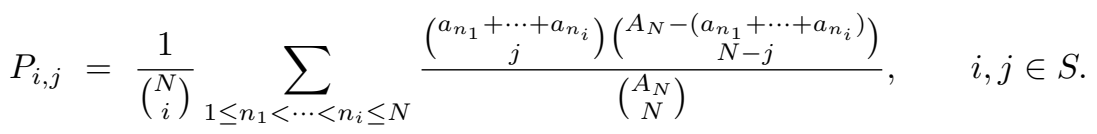

Note that $\mu$ has joint factorial moments

$$
\mathbb{E}\left(\left(\begin{array}{c}
\mu_{1} \\
l_{1}
\end{array}\right) \cdots\left(\begin{array}{c}
\mu_{N} \\
l_{N}
\end{array}\right)\right)=\frac{\left(\begin{array}{c}
N \\
l_{1}+\cdots+l_{N}
\end{array}\right)}{\left(\begin{array}{c}
A_{N} \\
l_{1}+\cdots+l_{N}
\end{array}\right)}\left(\begin{array}{c}
a_{1} \\
l_{1}
\end{array}\right) \cdots\left(\begin{array}{c}
a_{N} \\
l_{N}
\end{array}\right), \quad l_{1}, \ldots, l_{N} \in \mathbb{N}_{0},
$$

In particular, $\mathbb{E}\left(\mu_{n}\right)=N a_{n} / A_{N}, n \in\{1, \ldots, N\}$. From (12), it follows that the backward process $\widehat{X}$ has transition probabilities

$$
\widehat{P}_{i, j}=\frac{1}{\left(\begin{array}{c}
A_{N} \\
i
\end{array}\right)} \sum_{1 \leq n_{1}<\cdots<n_{j} \leq N} \sum_{\substack{l_{1}, \ldots, l_{j} \in \mathbb{N} \\
l_{1}+\cdots+l_{j}=i}}\left(\begin{array}{c}
a_{n_{1}} \\
l_{1}
\end{array}\right) \cdots\left(\begin{array}{c}
a_{n_{j}} \\
l_{j}
\end{array}\right), \quad i, j \in S,
$$

with the convention that $\widehat{P}_{i, 0}=\delta_{i 0}, i \in S$. Note that $\widehat{P}_{i, 1}=\left(\left(A_{N}\right)_{i}\right)^{-1} \sum_{n=1}^{N}\left(a_{n}\right)_{i}$, $i \in\{1, \ldots, N\}$, and, hence, $c_{N}=\widehat{P}_{2,1}=\left(\left(A_{N}\right)_{2}\right)^{-1} \sum_{n=1}^{N}\left(a_{n}\right)_{2}$ and $d_{N}=\widehat{P}_{3,1}=$ 
$\left(\left(A_{N}\right)_{3}\right)^{-1} \sum_{n=1}^{N}\left(a_{n}\right)_{3}$. The coalescence probability $c_{N}$ can be smaller than $1 / N$ (choose for example $a_{n}=2$ for all $n \in \mathbb{N}$ leading to $c_{N}=1 /(2 N-1)$ ), so the effective population size $N_{e}=1 / c_{N}$ can be larger than $N$. We have $c_{N}=0$ if and only if $a_{n}=1$ for all $n \in\{1, \ldots, N\}$. For the rest of this section it is assumed that $a_{n}>1$ for at least one index $n \in \mathbb{N}$ such that $c_{N}>0$ for sufficiently large $N$. In the following it is verified that $c_{N} \rightarrow 0$ if and only if $d_{N} / c_{N} \rightarrow 0$. From $\left(a_{n}-2\right) /\left(A_{N}-2\right) \leq\left(a_{n}-1\right) /\left(A_{N}-1\right) \leq a_{n} / A_{N}$ it follows that

$$
\max _{1 \leq n \leq N}\left(\frac{a_{n}-2}{A_{N}-2}\right)^{2} \leq \max _{1 \leq n \leq N} \frac{a_{n}\left(a_{n}-1\right)}{A_{N}\left(A_{N}-1\right)} \leq \sum_{n=1}^{N} \frac{a_{n}\left(a_{n}-1\right)}{A_{N}\left(A_{N}-1\right)}=c_{N} .
$$

Thus, $\max _{1 \leq n \leq N}\left(\left(a_{n}-2\right) /\left(A_{N}-2\right)\right) \leq c_{N}^{1 / 2}$, and consequently

$$
\begin{aligned}
d_{N} & =\sum_{n=1}^{N} \frac{a_{n}\left(a_{n}-1\right)\left(a_{n}-2\right)}{A_{N}\left(A_{N}-1\right)\left(A_{N}-2\right)} \\
& \leq\left(\max _{1 \leq n \leq N} \frac{a_{n}-2}{A_{N}-2}\right) \sum_{n=1}^{N} \frac{a_{n}\left(a_{n}-1\right)}{A_{N}\left(A_{N}-1\right)}=\left(\max _{1 \leq n \leq N} \frac{a_{n}-2}{A_{N}-2}\right) c_{N} \leq c_{N}^{3 / 2},
\end{aligned}
$$

or, equivalently, $d_{N} / c_{N} \leq c_{N}^{1 / 2}$. Therefore, if $c_{N} \rightarrow 0$, then $d_{N} / c_{N} \rightarrow 0$. As already mentioned in Section 2, the converse holds for arbitrary Cannings models, so if $d_{N} / c_{N} \rightarrow 0$, then $c_{N} \rightarrow 0$. In particular, the model is in the domain of attraction of the Kingman coalescent if and only if $c_{N} \rightarrow 0$ as $N \rightarrow \infty$.

Example 7.1. (power law growth) Fix $K \in \mathbb{N}_{0}$ and suppose that $a_{n}=n^{K}, n \in \mathbb{N}$. Then, $A_{N}=\sum_{n=1}^{N} n^{K} \sim N^{K+1} /(K+1)$ and, hence,

$$
c_{N}=\frac{1}{\left(A_{N}\right)_{2}} \sum_{n=1}^{N}\left(n^{K}\right)_{2} \sim \frac{1}{A_{N}^{2}} \sum_{n=1}^{N} n^{2 K} \sim\left(\frac{K+1}{N^{K+1}}\right)^{2} \frac{N^{2 K+1}}{2 K+1}=\frac{(K+1)^{2}}{2 K+1} \frac{1}{N} \text {. }
$$

Thus $N_{e}=\rho N$ with $\rho:=(2 K+1) /(K+1)^{2}<1$. Similarly,

$$
d_{N} \sim \frac{1}{A_{N}^{3}} \sum_{n=1}^{N} n^{3 K} \sim\left(\frac{K+1}{N^{K+1}}\right)^{3} \frac{N^{3 K+1}}{3 K+1}=\frac{(K+1)^{3}}{3 K+1} \frac{1}{N^{2}} .
$$

In particular, $c_{N} \rightarrow 0$, so the model is in the domain of attraction of the Kingman coalescent. This example essentially coincides with Example 5.1 (i) with $\alpha:=-K$.

Example 7.2. (geometric growth) Fix $\lambda \in\{2,3, \ldots\}$ and suppose that $a_{n}=\lambda^{n}$, $n \in \mathbb{N}$. Then, $A_{N}=\sum_{n=1}^{N} \lambda^{n}=\lambda\left(\lambda^{N}-1\right) /(\lambda-1) \sim \lambda^{N+1} /(\lambda-1)$. Now proceed as in Example 5.2 with $\lambda>1$. Define $b:=1 / \lambda$ and $p_{n}:=(1-b) b^{n-1}=(\lambda-1) / \lambda^{n}$, $n \in \mathbb{N}$. For $l_{1}, \ldots, l_{j} \in \mathbb{N}$, it follows that

$$
\begin{aligned}
\Phi_{j}\left(l_{1}, \ldots, l_{j}\right) & =\frac{1}{\left(A_{N}\right)_{i}} \sum_{\substack{n_{1}, \ldots, n_{j}=1 \\
\text { all distinct }}}^{N}\left(a_{n_{1}}\right)_{l_{1}} \cdots\left(a_{n_{j}}\right)_{l_{j}} \\
& \sim \frac{1}{A_{N}^{i}} \sum_{\substack{n_{1}, \ldots, n_{j}=1 \\
\text { all distinct }}}^{N} \lambda^{n_{1} l_{1}+\cdots+n_{j} l_{j}} \rightarrow \sum_{\substack{n_{1}, \ldots, n_{j} \in \mathbb{N} \\
\text { all distinct }}} p_{n_{1}}^{l_{1}} \cdots p_{n_{j}}^{l_{j}},
\end{aligned}
$$


where the proof of the last convergence works as in Example 5.2. The measure $\Xi$ of the limiting $\Xi$-coalescent assigns its total mass $\Xi(\Delta)=(\lambda-1) /(\lambda+1)$ to the single point $p=\left(p_{1}, p_{2}, \ldots\right) \in \Delta$.

Acknowledgement. This work benefited from the support of the ANR MANEGE (ANR-09-BLAN-0215) headed by Sylvie Méléard. The authors furthermore acknowledge the support of the University of Cergy-Pontoise. The second author in particular thanks the Laboratoire de Physique Théorique et Modélisation at the University of Cergy-Pontoise for their kind hospitality during a one month visit in 2010, where most of this work was done.

\section{REFERENCES}

[1] Berestycki, N.; Pitman, J. (2007). Gibbs distributions for random partitions generated by a fragmentation process. J. Stat. Phys. 127, 381-418.

[2] Caballero, A. (1994). Developments in the prediction of effective population size. Heredity 73, 657-679.

[3] Cannings, C. (1974). The latent roots of certain Markov chains arising in genetics: a new approach. I. Haploid models. Adv. Appl. Probab. 6, 260-290.

[4] Cannings, C. (1975). The latent roots of certain Markov chains arising in genetics: a new approach. II. Further haploid models. Adv. Appl. Probab. 7, 264-282.

[5] Comtet, L. (1970). Analyse combinatoire. Tomes 1 et 2. Presses Universitaires de France, Paris.

[6] Eldon, B.; Wakeley, J. (2006). Coalescent processes when the distribution of offspring number of individuals is highly skewed. Genetics 172, 2621-2633.

[7] Ethier, S. N.; Kurtz, T. G. (1986). Markov Processes. Characterization and Convergence. Wiley, New-York.

[8] Ewens, W. J. (1972). The sampling theory of selectively neutral alleles. Theor. Pop. Biol. 3, 87-112.

[9] Gladstien, K. (1978). The characteristic values and vectors for a class of stochastic matrices arising in genetics. SIAM J. Appl. Math. 34, 630-642.

[10] Handa, K. (2010). The two-parameter Poisson-Dirichlet point process. Bernoulli 15, 10821116.

[11] Huillet, T.; Martinez, S. (2008). Dirichlet-Kingman partition revisited. Far East Journal of Theoretical Statistics 24, 1-33.

[12] Karlin, S.; McGregor, J. (1964). Direct product branching processes and related Markov chains. Proc. Nat. Acad. Sci. U.S.A. 51, 598-602.

[13] Karlin, S.; McGregor, J. (1965). Direct product branching processes and related Markov chains. I. Calculations of rates of approach to homozygosity. Proc. Internat. Res. Sem., Springer, Berlin, pp. 111-145.

[14] Kingman, J. F. C. (1982). The coalescent. Stoch. Process. Appl. 13, 235-248.

[15] Kolchin, V. F. (1986). Random Mappings. Translation Series in Mathematics and Engineering. Optimization Software Inc. Publications Division, New York.

[16] Liggett, T. M. (1985). Interacting particle systems. Grundlehren der Mathematischen Wissenschaften [Fundamental Principles of Mathematical Sciences] 276. Springer-Verlag, New York.

[17] Maruyama, T. (1977). Stochastic problems in population genetics. Lecture Notes in Biomathematics 17. Springer-Verlag, Berlin-New York.

[18] McSweeney, J. K.; Pittel, B. G. (2008). Expected coalescence time for a nonuniform allocation process. Adv. Appl. Probab. 40, 1002-1032.

[19] Möhle, M. (1998). Robustness results for the coalescent. J. Appl. Probab. 35, 438-447.

[20] Möhle, M. (1999). The concept of duality and applications to Markov processes arising in neutral population genetics models. Bernoulli 5, 761-777.

[21] Möhle, M. (2000). Total variation distances and rates of convergence for ancestral coalescent processes in exchangeable population models. Adv. Appl. Probab. 32, 983-993. 
[22] Möhle, M. (2010). Asymptotic results for coalescent processes without proper frequencies and applications to the two-parameter Poisson-Dirichlet coalescent. Stoch. Process. Appl. 120, 2159-2173.

[23] Möhle, M. (2010). Looking forwards and backwards in the multi-allelic neutral Cannings population model. J. Appl. Probab. 47, 713-731.

[24] Möhle, M.; Sagitov, S. (2001). A classification of coalescent processes for haploid exchangeable population models. Ann. Probab. 29, 1547-1562.

[25] Möhle, M.; Sagitov, S. (2003). Coalescent patterns in diploid exchangeable population models. J. Math. Biol. 47, 337-352.

[26] Moran, P. A. P.; Watterson, G. A. (1959). The genetic effects of family structure in natural populations. Austral. J. Biol. Sci. 12, 1-15.

[27] Pitman, E. J. G. (1968). On the behaviour of the characteristic function of a probability distribution in the neighbourhood of the origin. J. Austral. Math. Soc. 8, 422-443.

[28] Pitman, J. (2006). Combinatorial Stochastic Processes. Lecture Notes in Mathematics 1875, $13-35$.

[29] Sagitov, S. (2003). Convergence to the coalescent with simultaneous multiple mergers. $J$. Appl. Probab. 40, 839-854.

[30] Sagitov, S.; Jagers, P. (2005). The coalescent effective size of age-structured populations. Ann. Appl. Probab. 15, 1778-1797.

[31] Schweinsberg, J. (2000). Coalescents with simultaneous multiple collisions. Electron. J. Probab. 5, Paper no. 12, 50 pp.

[32] Schweinsberg, J. (2003). Coalescent processes obtained from supercritical Galton-Watson processes. Stoch. Process. Appl. 106, 107-139.

[33] Steutel, F. W. and van Harn, K. (1979). Discrete analogues of self-decomposability and stability. Ann. Probab. 7, 893-899.

[34] Wakeley, J.; Sargsyan, O. (2009). Extensions of the coalescent effective population size. Genetics 181, 341-345.

\section{THIERRY HUILLET}

Laboratoire de Physique Théorique et Modélisation

CNRS-UMR 8089 et Université de Cergy-Pontoise

2 Avenue Adolphe Chauvin

95302 CERGy-Pontoise, FRANCE

E-MAIL: THIERRY.HUILLET@U-CERGY.FR

Martin MÖHLE

MATHEMATISChES Institut

EBERHARD Karls Universität TÜBINGEN

Auf Der Morgenstelle 10

72076 TÜBINGEN, GERMANY

E-MAIL: MARTIN.MOEHLE@UNI-TUEBINGEN.DE 